\title{
Optical properties and chemical ordering of Ag-Pt nanoalloys: a computational study
}

\author{
Sofia Olobardi, ${ }^{\dagger}$ Alessandro Fortunelli, ${ }^{\S}$ Mauro Stener ${ }^{*}, \dagger$ \\ Francesc Viñes, ${ }^{\star}$ Lorena Vega ${ }^{\ddagger}$ and Konstantin M. Neyman ${ }^{*}+$, ,
}

${ }^{\dagger}$ Dipartimento di Scienze Chimiche e Farmaceutiche, Università di Trieste, via L. Giorgieri 1, I34127, Trieste, Italy

* Departament de Ciència de Materials i Química Física \& Institut de Química Teòrica i

Computacional (IQTCUB), Universitat de Barcelona, Martí i Franquès 1-11, 08028 Barcelona, Spain

${ }^{\S}$ CNR-ICCOM \& IPCF, Consiglio Nazionale delle Ricerche, via G. Moruzzi 1, 56124, Pisa, Italy

"ICREA (Institució Catalana de Recerca i Estudis Avançats), 08010 Barcelona, Spain

\begin{abstract}
A series of core@shell and layered ordered phases of AgPt bimetallic nanoparticles has been studied with $\mathrm{Ag}: \mathrm{Pt}=3: 1$ and 6:1 atomic compositions and sizes from 116 to 201 atoms. The elementary chemical order has been established by using a recent method (TOP), which assigns energy according to different topological degrees of freedom. The TOP lowest-energy structures, confirmed by density functional calculations, are then studied by time dependent density functional theory in order to calculate optical properties. The present study shows that for $\mathrm{AgPt}$ nanoparticles with core@shell structure the optical properties are sensitive to both the Pt concentration and system size. Spectral trends related to chemical order have also been identified.
\end{abstract}

\footnotetext{
*Corresponding authors’ emails: stener@units.it; konstantin.neyman@icrea.cat
} 


\section{Introduction}

Metal nanoparticles (NPs) are intriguing systems, which exhibit specific behaviors connected with their nanoscopic nature and are important for both basic sciences and technological applications. From the structural point of view, the atomic arrangement of nanoparticles can differ with respect to the corresponding bulk materials, due to surface tension effects. In the case of nanoalloys, their properties may depend on the metal concentration as well as on the chemical ordering, offering many possibilities with increasing complexity. Theoretical methods are important and widely employed in order to rationalize the observed behaviors, with the challenging aim to design new materials with given properties. ${ }^{1}$ While studying nanoalloys the computational problems are two-fold: on one side an efficient and accurate method to calculate optical properties becomes necessary, and, on the other hand, the structure, composition, and chemical ordering should be reliably modelled in order to calculate the optical properties. From the structural point of view the most challenging problem to describe nanoalloys is the chemical ordering. In fact the number of homotops (i.e. topological isomers) for a given chemical composition and shape of a nanoparticle is so high that a simplified approach must be invoked to make the problem of this extraordinarily high dimensionality tractable in practice.

In this work the chemical ordering problem has been tackled by means of the TOP method, a novel, efficient, and versatile computational tool for determining the atomic arrangement in bimetallic nanoparticles with different combinations of metals in a wide range of sizes, shapes, and compositions. ${ }^{2}$ The original methodology allowed obtaining homotops with, $a$ priori, the most energetically stable chemical ordering corresponding to the ground state of bare species at low temperature. A recent implementation ${ }^{2,3}$ enables dealing with representative nanoparticles models of experimental structures also at elevated temperatures, and is efficiently and accurately applicable to both high- and low-symmetry particles. The interest in such a methodology arises from the observation that in bimetallic NPs atoms of one element may have a preference for the interior or surface sites, as well as for the formation of heteroatomic bonds or layered structures during the alloying process. ${ }^{4,5}$ The additional degrees of freedom that bimetallic nanocrystals exhibit compared to monometallic counterparts can considerably affect their structural complexity, and so their properties. Furthermore, an exact experimental structural characterization is still limited, due to the absence of an adequate three-dimensional atomic resolution standard setups. On the other hand, electronic structure calculations are explanatory and have displayed predictive power. However, characterizing atomic arrangements of even moderately large heterometallic nanoparticles by means of simulations is still a challenging task. 
Once the structures of the systems and their chemical ordering are assessed, it is possible to calculate their optical properties. In this respect the best compromise between accuracy and computational expenditures is offered by the Time Dependent Density Functional Theory (TDDFT) formalism. In the present work we employ a recent scheme to solve the TDDFT equations representing the induced density over an auxiliary basis set of Slater Type Orbitals (STO $)^{6-8}$ which has proven to be very efficient to treat quite large metal particles containing up to several hundred atoms. 9

In the photoabsorption experiments it has been found that while pure Ag clusters display strong Surface Plasmon Resonance (SPR), such feature disappears in bimetallic Ag-Pt nanoclusters, unless a very large cluster size (around $10 \mathrm{~nm}$ ) is reached. ${ }^{10}$ The role of Pt in the SPR suppression in AgPt clusters has been already considered in a previous work. ${ }^{11}$ The present work deals with AgPt nanoalloys of different size and composition mixing silver and platinum atoms. Specifically, three different types of nanoparticles composed of 116, 140, or 201 atoms have been studied. For each size, an Ag-rich situation has been investigated, with $\mathrm{Ag}: \mathrm{Pt}$ proportions of about 3:1 and 6:1 considering NPs with a truncated octahedron geometry. In addition, particles with a layered structure and Ag:Pt composition ratio ca. 4:1 have been considered as well, since a recent experiment has demonstrated the existence of AgPt multilayer nanoparticles with a $\mathrm{L}_{1}$ phase coated by a monoatomic Ag skin. ${ }^{12}$

$\mathrm{AgPt}$ nanoparticles are used in several catalytic processes; ${ }^{13}$ as an example, $\mathrm{H}_{2} \mathrm{O}_{2}$ production by direct $\mathrm{H}_{2}$ oxidation synthesis $\left(\mathrm{H}_{2}+\mathrm{O}_{2} \rightarrow \mathrm{H}_{2} \mathrm{O}_{2}\right)$ is a promising alternative to replace common chlorinated industrial oxidants. A reaction rate of approximately 10 -fold greater on AgPt NPs than on Pt NPs of similar size ${ }^{14,15}$ showed that AgPt nanoparticles deposited on multi-walled carbon nanotubes (MWCNTs) exhibit a higher electrocatalytic activity for oxygen reduction. ${ }^{15}$ Interestingly, the localized surface plasmon resonance excitation in plasmonic NPs has been used to accelerate catalytic transformations under visible-light irradiation. ${ }^{16}$ AgPt NPs with different size, shape, and composition have been synthesized and characterized, as there are experimental evidences indicating existence of both core@shell structures ${ }^{13}$ and multilayers with $\mathrm{L} 1_{1}$ phase coated by a monoatomic Ag skin. ${ }^{12}$

\section{Theory}

\section{Structures and homotops: the TOP method}

The TOP method ${ }^{2}$ is based on the analysis of the energy related to topological degrees of freedom, called $E_{T O P}$ (see Equation 1). In the case of a bimetallic NP with a predefined $\mathrm{A}_{\mathrm{n}} \mathrm{B}_{\mathrm{m}}$ 
composition and crystalline lattice, the energy difference between any two chosen homotops is considered to depend only on the respective mutual positions of $\mathrm{A}$ and $\mathrm{B}$ atoms:

$\Delta E_{\mathrm{TOP}}=\varepsilon_{B O N D}^{A-B} \cdot N_{B O N D}^{A-B}+\varepsilon_{C O R N E R}^{A} \cdot N_{C O R N E R}^{A}+\varepsilon_{E D G E}^{A} \cdot N_{E D G E}^{A}+\varepsilon_{T E R R A C E}^{A} \cdot N_{T E R R A C E}^{A}$

namely, on the number of A-B bonds (nearest-neighbor pairs of atoms $\mathrm{A}$ and $\mathrm{B}, N_{B O N D}^{A-B}$ ) and the numbers of corner $\left(N_{C O R N E R}^{A}\right)$, edge $\left(N_{E D G E}^{A}\right)$ and terrace $\left(N_{T E R R A C E}^{A}\right)$ surface A atoms in the homotops under scrutiny. Other variables such as $N_{B O N D}^{A-A}, N_{B O N D}^{B-B}, N_{C O R N E R}^{B}, N_{E D G E}^{B}, N_{T E R R A C E}^{B}$ and $N_{I N T E R I O R}^{A}$ that can depend on the employed variables are not included in the simplest $E_{T O P}$ expression (1). The $\varepsilon_{i}^{X}$ are energetic variables associated with each degree of freedom, $N_{i}^{X}$, contributing to the topological energy, called descriptors. In contrast to parameters in many empirical methods, each descriptor $\varepsilon_{i}^{X}$ has a clear physical meaning.

The descriptors in the $E_{T O P}$ energy expression (Eq. 1) for each NP size and composition are derived from a rigorous fitting procedure based on energies gained on a limited set of density functional (electronic structure) calculations. The electronic structure calculations were performed using the periodic plane-wave code VASP (Vienna Ab Initio Simulation Package) ${ }^{17}$ with the Perdew-Burke-Ernzerhof (PBE) ${ }^{18}$ exchange-correlation functional. The PBE exchangecorrelation functional was found to be one of the most appropriate among common functionals to describe transition metal bulks and surfaces. ${ }^{19-21}$ The interaction between valence and core electrons was treated within the PAW (Projector Augmented Wave) approach. In order to moderate the computational cost, a $275 \mathrm{eV}$ energy cutoff for the plane-wave basis sets was used. The one-electron levels were smeared by $0.1 \mathrm{eV}$ using the first-order method of Methfessel and Paxton, ${ }^{22}$ and the converged energies were extrapolated to zero smearing. All calculations were performed only at the $\Gamma$-point in the reciprocal space, and the full set of atoms was allowed to locally relax during the geometry optimization. These electronic structure calculations represent the most challenging, computationally speaking, part of the method. To reduce computational costs, the minimal separation between NPs was chosen to be $>0.7 \mathrm{~nm}$, a typical safe value which guarantees negligible interaction between adjacent NPs in neighboring supercells. ${ }^{23}$

\section{Optical properties: the TDDFT complex polarizability}

The ADF (Amsterdam Density Functional) program has been used to calculate the optical properties at the TDDFT level. The complex polarizability method has been employed to solve the TDDFT equations as described previously, ${ }^{6,7}$ so the reader is referred to the original papers for detailed information on the methodology and numerical implementation. The PBE exchangecorrelation (xc) functional 18 is chosen to solve the KS equations while the Adiabatic Local Density Approximation ${ }^{24}$ (ALDA) is used in the TDDFT part for the exchange-correlation 
kernel. The PBE xc-functional has been chosen because the LB94, ${ }^{25}$ which should be more suitable for TDDFT since it supports the correct Coulomb tail, has proven not to be appropriate for AgPt clusters. ${ }^{11}$ The SAOP xc-functional ${ }^{26}$ would have been computationally too expensive due to the necessity of all-electron basis set. A basis set of STO included in the ADF database of triple-zeta polarized (TZP) quality for Pt and double-zeta (DZ) quality for Ag with frozen core (up to Pt $4 f$ and $\mathrm{Ag} 4 p$ ) have been employed. Relativistic effects (which are important for heavy elements such as platinum) have been treated at the Zero Order Regular Approximation (ZORA) level. ${ }^{27}$

\section{Results}

\section{Structures}

The $\varepsilon_{i}$ descriptors were determined by fitting $E_{T O P}$ values of Eq. 1 to the total energies $E_{D F T}$ of series of homotops of the optimized NP structures under scrutiny via multiple linear regression. These descriptor values were used to optimize the chemical ordering in the NPs at the DFT level by optimizing lowest-energy homotops predicted by means of Monte-Carlo simulation applying Eq. 1 as detailed elsewhere. ${ }^{2,3}$ For each NP, 73 different homotops were used for the fitting procedure. The resulting TOP descriptors (in $\mathrm{eV}) \varepsilon_{B O N D}^{P t-A g}, \varepsilon_{C O R N E R}^{P t}, \varepsilon_{E D G E}^{P t}, \varepsilon_{T E R R A C E}^{P t}$ for the studied by DFT six AgPt NPs are as follows, respectively: 0.015, 0.494, 0.518, $0.236\left(\mathrm{Ag}_{87} \mathrm{Pt}_{29}\right)$; 0.004, 0.434, 0.468, $0.311\left(\mathrm{Ag}_{99} \mathrm{Pt}_{17}\right) ; 0.004,0.418,0.272,0.198\left(\mathrm{Ag}_{105} \mathrm{Pt}_{35}\right)$; -0.004, 0.483, 0.245, $0.177\left(\mathrm{Ag}_{120} \mathrm{Pt}_{20}\right) ; 0.010,0.601,0.307,0.207\left(\mathrm{Ag}_{151} \mathrm{Pt}_{50}\right) ; 0.007,0.320,0.252,0.232$ $\left(\mathrm{Ag}_{172} \mathrm{Pt}_{29}\right)$.

Since the descriptors $\left\{\varepsilon_{i}\right\}$ have an intrinsic well-defined physical origin, their values allow directly rationalizing the nature of binding in the considered alloys. For example $\varepsilon_{B O N D}^{A-B}$ is related to the energy gain caused by the bonding of two different metal atoms A and B in the NP. According to the obtained results, for different studied nanoparticles there is only a quite moderate variation upon the specific descriptor values. In particular, the energy gain due to the formation of heteroatomic bonds is rather small for these nanomaterials, and so it plays a secondary role in the determination of the NP ordering. In all cases, the stability of Pt atoms is highest inside the NP and lowest in the NP surface sites - corners, edges, and terraces. In general, the less the site is coordinated, the higher is the energy of a Pt atom in that position. This effect leads to the core@shell-like structure of the lowest-energy AgPt homotops, which have the surface shell enriched with Ag and the core composed mostly of Pt. In Figure 1 the optimized structures of all lowest-energy homotops under scrutiny (putative global minima) are shown. Studies on bimetallic nanoparticles ${ }^{2,5}$ composed of PdAu and PdAg revealed similar trends in 
the chemical ordering, defining that the coinage metal atoms Au and Ag preferentially occupy positions with lower coordination numbers in core@shell structures.

\section{Optical properties}

For each of the six AgPt clusters, a TDDFT photoabsorption spectrum was calculated (see Figure 2). The charge of the clusters has been chosen in order to obtain a closed@shell electronic structure. In some instances, a closed@shell electronic structure can be obtained for different values of the cluster charge. For example, $\left[\mathrm{Ag}_{99} \mathrm{Pt}_{17}\right]^{+}$and $\left[\mathrm{Ag}_{99} \mathrm{Pt}_{17}\right]^{-}$are both closed shell and the effect of the charge on the optical photoabsorption spectrum is considered in Figure S1 of the Supporting Information (SI). The effect is quite modest, although not so small as in pure gold or silver clusters. ${ }^{28}$ The general shape of the photoabsorption remains very similar for the two charge states, with a regular intensity increasing from $2 \mathrm{eV}$ up to $5 \mathrm{eV}$, and with an evident feature around $4.5 \mathrm{eV}$ in both cases. Then the intensity is modulated but remains constant in average up to $7 \mathrm{eV}$ when it starts to decrease.

Figure 2 displays the calculated spectra together with each partial contribution split according to the Cartesian component of the electric dipole operator. In all cases all partial contributions are almost superimposable, confirming an isotropic absorption as an effect of approximatively spherical distribution of the Pt dopant within the Ag cluster. In Figure 3 the clusters are grouped in order to analyze the photoabsorption trend with respect to increasing cluster size keeping constant Ag:Pt chemical compositions.

The upper panel of Figure 3 combines spectra for the clusters with the composition $\mathrm{Ag}: \mathrm{Pt}$ $=3: 1\left[\mathrm{Ag}_{87} \mathrm{Pt}_{29}\right]^{-},\left[\mathrm{Ag}_{105} \mathrm{Pt}_{35}\right]^{3+}$, and $\left[\mathrm{Ag}_{151} \mathrm{Pt}_{50}\right]^{+}$while the lower panel shows spectra for the clusters with the composition $\mathrm{Ag}: \mathrm{Pt}=6: 1\left[\mathrm{Ag}_{99} \mathrm{Pt}_{17}\right]^{+}, \mathrm{Ag}_{120} \mathrm{Pt}_{20}$, and $\left[\mathrm{Ag}_{172} \mathrm{Pt}_{29}\right]^{4+}$. In both cases as the size increases the intensity obviously increases, and the spectral features essentially keep their energy positions as well their intensity with respect to the background.

For the Ag:Pt = 3:1 composition the size effect is rather weak for the two smallest clusters, but it is much more pronounced going from $\left[\mathrm{Ag}_{105} \mathrm{Pt}_{35}\right]^{3+}$ to $\left[\mathrm{Ag}_{151} \mathrm{Pt}_{50}\right]^{+}$. Interestingly, for the $\mathrm{Ag}: \mathrm{Pt}=6: 1$ composition the size effect is of the same magnitude when going from $\left[\mathrm{Ag}_{99} \mathrm{Pt}_{17}\right]^{+}$to $\mathrm{Ag}_{120} \mathrm{Pt}_{20}$ as when going from $\mathrm{Ag}_{120} \mathrm{Pt}_{20}$ to $\left[\mathrm{Ag}_{172} \mathrm{Pt}_{29}\right]^{4+}$. The behavior for low $\mathrm{Pt}$ concentration is therefore smooth. This suggests that Pt doping at higher concentrations is less regular, so at small size the $\mathrm{Pt}$ doping is more effective while at larger size the plasmon collective effects of silver become more pronounced.

In Figure 4 the clusters with the same size but different Pt concentrations are grouped together in order to study the effect of the chemical composition on the photoabsorption. Starting the comparison with the smallest pair $\left(\left[\mathrm{Ag}_{87} \mathrm{Pt}_{29}\right]^{-}\right.$and $\left.\left[\mathrm{Ag}_{99} \mathrm{Pt}_{17}\right]^{+}\right)$we notice that when the $\mathrm{Pt}$ 
concentration increases the intensity is increased up to $6 \mathrm{eV}$, while from $6 \mathrm{eV}$ to $7 \mathrm{eV}$ the intensity is quite comparable and above $7 \mathrm{eV}$ the intensity is lower at higher Pt concentration.

A very useful tool to discuss the spectra and their trends in nanoalloys is the analysis in terms of fragments ${ }^{29}$ as reported in Figure 5 for all studied systems. In fact for the present nanoalloys it is very natural to define two fragments, each one consisting of the atoms of the same element. With this tool the photoabsorption profile is split in four components, giving the weight of the fragment in initial state and final state, so in the figure are displayed these four contributions, namely, $\mathrm{Ag} \rightarrow \mathrm{Ag}, \mathrm{Ag} \rightarrow \mathrm{Pt}, \mathrm{Pt} \rightarrow \mathrm{Ag}$, and $\mathrm{Pt} \rightarrow \mathrm{Pt}$ using different colors. The fragment analysis for $\left[\mathrm{Ag}_{87} \mathrm{Pt}_{29}\right]^{-}$, shows that up to $5 \mathrm{eV} \mathrm{Pt} \rightarrow \mathrm{Ag}$ is the most important contribution, while $\mathrm{Ag} \rightarrow \mathrm{Ag}$ becomes the leading one at higher energies. The other contributions are less relevant, in particular $\mathrm{Pt} \rightarrow \mathrm{Pt}$ one is weak but rather constant over the whole considered energetic interval, while $\mathrm{Ag} \rightarrow \mathrm{Pt}$ one is weak but follows the shape of $\mathrm{Ag} \rightarrow \mathrm{Ag}$, although strongly reduced. The fragment analysis for this system reveals that the silver absorption plays a major role at high energy $(>5 \mathrm{eV})$, however at lower energy the importance of the $\mathrm{Pt} \rightarrow \mathrm{Ag}$ contributions suggests that the absorption can be described as an inter-band transition from occupied valence Pt states to virtual Ag empty states. In summary, Pt doping can be described as an effect of increasing the availability of electrons, which can be easily promoted to silver by light absorption.

It is interesting to analyze in more detail two most salient spectral features of $\left[\mathrm{Ag}_{87} \mathrm{Pt}_{29}\right]^{-}$, which are the absorption maxima at $4.32 \mathrm{eV}$ and $5.16 \mathrm{eV}$, respectively. From the fragment analysis the first band must be ascribed essentially to a $\mathrm{Pt} \rightarrow \mathrm{Ag}$ transition, while the second one is contributed by both $\mathrm{Ag} \rightarrow \mathrm{Ag}$ and $\mathrm{Pt} \rightarrow \mathrm{Ag}$ transitions. This analysis allows identifying the spatial region involved in the transition, but it is not able to describe the transition in terms of electronic structure or ascribe a specific nature to the transition, for example, if a plasmonic behavior is present or not. A further and powerful analysis tool is the Individual Component Map of the Oscillator Strength ${ }^{30}$ (ICM-OS), which allows ascribing a specific absorption peak to a collection of occupied-virtual pairs, which contribute to the oscillator strength. ICM-OS is very useful since it takes into account not only the weight of the pairs, but also the dipole contribution, so also negative contributions are possible due to destructive interference among excited configurations. The ICM-OS analysis of the $4.32 \mathrm{eV}$ and $5.16 \mathrm{eV}$ features of $\left[\mathrm{Ag}_{87} \mathrm{Pt}_{29}\right]^{-}$, is presented in Figure 6: in the $4.32 \mathrm{eV}$ peak we observe three strong spots on the diagonal (the diagonal corresponds to an energy difference between virtual and occupied orbitals equal to the excitation energy) together with strong extra-diagonal spots parallel to the occupied orbitals energy axis, corresponding to lower energy configurations with final states close to the LUMO. 
This shape is typically plasmonic, since the presence of extra-diagonal spots is an indication of a collective behavior. ICM-OS analysis of the peak at $5.16 \mathrm{eV}$ provides a much simpler picture: almost all the intensity is carried by a single strong spot on the diagonal, ruling out the presence of a plasmon. This finding is quite surprising, since the $5.16 \mathrm{eV}$ peak is of pure silver nature according to previous fragment analysis, so it would have been rather natural to ascribe plasmonic behavior to such silver peak. On the other hand, the fragment nature of the $4.16 \mathrm{eV}$ plasmonic peak is essentially $\mathrm{Pt} \rightarrow \mathrm{Ag}$, with low silver participation in the initial states. Also this finding is interesting, since plasmonic behavior is expected to be limited to silver contributions.

In order to extrapolate Pt doping effect to zero, we have calculated a series of pure silver clusters (Figure S2) obtained by substituting Pt atoms in the present bimetallic clusters with Ag atoms, imposing charges required for closed@shell electronic structures. The spectra are characterized by an intense plasmonic peak just above $3 \mathrm{eV}$, which becomes narrower and stronger as the particle size increases. Comparing these spectral features with those of the bimetallic clusters we observe that in pure Ag clusters the photoabsorption starts suddenly around $3 \mathrm{eV}$, while in bimetallic clusters the photoabsorpion starts smoothly around $1 \mathrm{eV}$. Moreover, the spectral features well defined in Ag clusters become unresolved and just sketched<?> peaks over a smooth background in AgPt. In general, the presence of Pt reduces the plasmonic behavior and makes all the spectral features much less prominent.

The above discussion<analysis? $>$ about the plasmonic behavior of $\left[\mathrm{Ag}_{87} \mathrm{Pt}_{29}\right]^{-}$in terms of ICM-OS has been also performed for the two most intense features of all other clusters, see ICM-OS plots in Figures S3 and S4. The conclusions are consistent with those for $\left[\mathrm{Ag}_{87} \mathrm{Pt}_{29}\right]^{-}$: the lower-energy feature displays strong plasmonic character, which is lost in the feature at higher energy. It is interesting that for the lager clusters the low-energy peak is still plasmonic but its intensity is much lower than for pure Ag clusters (see Figure S2). This effect can be explained by noticing negative contributions in ICM-OS plots near the diagonal: such contributions give rise to a destructive interference strongly reducing the plasmon intensity.

Summarizing the results of the above analysis we can rationalize the plasmonic peak in the AgPt nanoalloys as follows: when Ag nanoparticles are doped by a small amount of Pt the plasmon keeps its Ag nature only as concerns the final states, which remain on silver. On the other hand, the initial states are mainly ascribed to Pt. This happens because Pt has an excess of electronic charge compared to Ag. This rationalization in terms of electronic structure is consistent with the Partial Density of States (PDOS) plot in Figure 7, where the energy scale is shifted with respect to Fermi energy taken as the zero energy. All NPs considered in this work show an intense PDOS in the range $-6 \mathrm{eV}-2 \mathrm{eV}$, which corresponds to the $4 d$ silver band. As the energy increases the $\mathrm{Ag}$ PDOS smoothly decreases and it becomes irregular but constant in 
average above the Fermi level. Differently, the Pt contribution is rather uniform and low on the whole energy scale, and it becomes negligible above the Fermi energy. The only region where the Pt contribution is comparable to that of $\mathrm{Ag}$ is just $2 \mathrm{eV}$ below the Fermi energy: there the Pt role is even more pronounced than of Ag. Overall, PDOS are dominated by silver states, the only region where Pt contribution is important is just below the Fermi energy. Electrons there are the most mobile, in line with the description of the plasmon in terms of transition from $\mathrm{Pt}$ to $\mathrm{Ag}$.

Going back to the chemical composition analysis in Figure 4, we note a similar behavior also for the pairs $\left[\mathrm{Ag}_{105} \mathrm{Pt}_{35}\right]^{3+}$ with $\mathrm{Ag}_{120} \mathrm{Pt}_{20}$ and $\left[\mathrm{Ag}_{151} \mathrm{Pt}_{50}\right]^{+}$with $\left[\mathrm{Ag}_{172} \mathrm{Pt}_{29}\right]^{4+}$. The fragment analysis in Figure 5 indicates also other interesting features. For example, going from the left to the right (reducing the $\mathrm{Pt}$ concentration) one sees a pronounced increase of the $\mathrm{Ag} \rightarrow \mathrm{Ag}$ contribution, while the other important contribution $\mathrm{Pt} \rightarrow \mathrm{Ag}$ is not affected and the minor components $\mathrm{Pt} \rightarrow \mathrm{Pt}$ and $\mathrm{Ag} \rightarrow \mathrm{Pt}$ are even more reduced. PDOS in Figure 7 shows that only the energy region from $-2 \mathrm{eV}$ to $0 \mathrm{eV}$ is sensitive to the nanoparticle nature, in particular, to the $\mathrm{Pt}$ concentration, which is able to populate this energy region becoming the most important contribution for the nanoalloys with $\mathrm{Ag}$ :Pt ratio around 3:1.

\section{Multilayer $L 1_{0}$ and $L 1_{1}$ ordered structures}

Recent experimental and computational investigation ${ }^{12}$ on AgPt NPs has shown formation of a defect-free $\mathrm{L}_{1}$ ordered phase inside a Ag skin in particles smaller $2.5 \mathrm{~nm}$. Such ordered phase consists in alternating $\mathrm{Ag}$ and $\mathrm{Pt}$ (111) planes. To estimate the layered structure effects on optical properties we investigated two systems with chemical composition $\mathrm{Ag}: \mathrm{Pt}=4: 1: \mathrm{Ag}_{161} \mathrm{Pt}_{40}$ featuring homometallic layers perpendicular to the (100) direction ( $\mathrm{L}_{0}$ arrangement) and $\mathrm{Ag}_{158} \mathrm{Pt}_{43}$ with the layers perpendicular to the (111) direction ( $\mathrm{L}_{1}$ arrangement), the latter modeling the experimentally observed structure. ${ }^{12}$ Two homotops of these NPs featuring typical core@shell ordering have been also considered as references (see structures of these four models in Figure 8). DFT energy shows that for the particle $\mathrm{Ag}_{161} \mathrm{Pt}_{40}$ the $\mathrm{L} 1_{0}$ layered structure is less stable than the core@shell one by $1.7 \mathrm{eV}$, while for the similarly large particle $\mathrm{Ag}_{158} \mathrm{Pt}_{43}$ the $\mathrm{L}_{1}$ layered structure is more stable than the core@shell one by $1.6 \mathrm{eV}$. Although this finding is consistent with the experiment, indicating a preference for the $\mathrm{L} 1_{1}$ ordered phase, the energy difference is so tiny (around $8 \mathrm{meV}$ per atom) that it is more reasonable to assume very similar stability of the $\mathrm{L} 1_{1}, \mathrm{~L} 1_{0}$ and core@shell structures. This is supported by another stability indicator, so-called excess energy ${ }^{31,32} E_{\text {exc }}\left(\mathrm{Ag}_{201-\mathrm{n}} \mathrm{Pt}_{\mathrm{n}}\right)=\left\{E\left(\mathrm{Ag}_{201-\mathrm{n}} \mathrm{Pt}_{\mathrm{n}}\right)-[(201-\mathrm{n}) / 201] E\left(\mathrm{Ag}_{201}\right)\right.$ $\left.-(\mathrm{n} / 201) E\left(\mathrm{Pt}_{201}\right)\right\} / 201$, which is (in meV) -59 $\left(\mathrm{Ag}_{158} \mathrm{Pt}_{43}, \mathrm{~L}_{1}\right),-40\left(\mathrm{Ag}_{161} \mathrm{Pt}_{40}, \mathrm{L1}_{0}\right),-60$ $\left(\mathrm{Ag}_{151} \mathrm{Pt}_{50}\right.$, core@shell) and -37 ( $\mathrm{Ag}_{172} \mathrm{Pt}_{29}$, core@shell). Therefore, it is very hard to 
discriminate such ordered phases by simple energetic analysis, and other properties such as optical ones could be useful to help in this respect. Figure 9 displays photoabsorption spectra of these four models, calculated with charges giving closed-shell electronic structures. For the core@shell ordering spectra in panels a) and b), as already mentioned, the partial dipole contributions are very similar to each other due to the spherical shape of the systems. Going to the $\mathrm{L} 1_{0}$ and $\mathrm{L} 1_{1}$ layered structures — panels c) and d) — we observe that the profile is much less smooth than that in the corresponding core@shell structures. Moreover, the Z component of the electric dipole contribution for the $\mathrm{L} 1_{0}$ structure displays features, which are out-of-phase with respect to the $\mathrm{X}$ and $\mathrm{Y}$ components. Both effects can be ascribed to the presence of layers which reduce the symmetry and increase anisotropy. It is worth to directly compare the spectra of core@shell and layered structures _ panels e) and f) — in order to easier identify the effect of the chemical order. For both NPs the effect is quite modest. At low energy (below $<$ above? $>3 \mathrm{eV}$ ) the layered structure promotes a weak increase of the intensity, a slightly more pronounced increase is apparent between 4 and $6 \mathrm{eV}$ for both NPs. Above $6 \mathrm{eV}$ chemical ordering has almost no effect for $\mathrm{Ag}_{158} \mathrm{Pt}_{43}$, while for $\mathrm{Ag}_{161} \mathrm{Pt}_{40}$ a slight decrease is observed. The rather modest effect of the chemical ordering is not surprising since in both structures the Pt atoms are confined inside the particles covered by a complete Ag skin. This suggests that the photoabsorption is localized on the surface, which is well established for plasmons. This is also consistent with a previous computational work ${ }^{11}$ on AgPt clusters, where the effect of $\mathrm{Pt}$ doping on optical properties was found more pronounced when Pt atoms were set on the surface.

Partial DOS plots of the core@shell and layered $\mathrm{Ag}_{158} \mathrm{Pt}_{43}$ and $\mathrm{Ag}_{161} \mathrm{Pt}_{40}$ models are shown in Figure S5. Although the global shape closely resembles that in Figure 7, Pt partial DOS in the layered models is strongly enhanced between 0 and $2 \mathrm{eV}$ below the Fermi energy. This is consistent with the weak absorption enhancement at low energy. The fragment analysis (Figure S6) agrees with previous data. Only the $\mathrm{Ag} \rightarrow \mathrm{Ag}$ partial contribution deserves a remark: while for the core@shell orderings it remains very low up to $4 \mathrm{eV}$, this contribution is more intense and structured between 2 and $4 \mathrm{eV}$ for the layered orderings. Also the ICM-OS analysis (Figure S7) of the core-shell and layered $\mathrm{Ag}_{158} \mathrm{Pt}_{43}$ particles taken at two energies (4.65 and $5.54 \mathrm{eV}$ ) corresponding to the most salient spectral features agrees with the previous analysis: only the peak at lower energy $(4.65 \mathrm{eV})$ displays a plasmonic behavior. It is worth noting, however, that the plasmonic behavior is enhanced in the layered model compared to the core-shell one.

\section{Conclusions}

The present study shows that for AgPt nanoclusters with core@shell structure, the optical properties are sensitive to both $\mathrm{Pt}$ concentration and the size. For low $\mathrm{Pt}$ concentration $(\mathrm{Ag}: \mathrm{Pt}=$ 
6:1) the size effect is smooth, while a less regular trend is identified when Pt concentration is higher $(\mathrm{Ag}: \mathrm{Pt}=3: 1)$. On the other hand, for constant cluster size, the Pt concentration is found to shift intensity from the low energy part (which is depleted up to around $6 \mathrm{eV}$ ) to the high energy region (above $7 \mathrm{eV}$ ) where intensity increases with Pt concentration. Such findings have been rationalized in terms of recently developed tools (fragment analysis and $<$ to spell-out again in the Conclusions $>$ ICM-OS). Fragment analysis suggests that the low energy part of the spectrum is dominated by the $\mathrm{Pt} \rightarrow \mathrm{Ag}$ contribution, while at higher energy $\mathrm{Ag} \rightarrow \mathrm{Ag}$ contribution plays a major role. ICM-OS analysis allows identifying collective behaviors and therefore to follow the evolution of the plasmon for different models.

Special attention has been devoted to clusters with ordered phase (in particular $\mathrm{L} 1_{1}$ one), which have been recently found in experiments for AgPt nanoparticles with size below $2.5 \mathrm{~nm}$. In this case rather modest differences with respect to core@shell structures have been found in the optical properties. This has been rationalized by the fact that the photoabsorption takes place mainly on the surface which is made of Ag atoms. In fact, also the studied ordered phases are covered by a monatomic silver skin due to the energetic destabilization of Pt atoms in all surface positions compared to inner ones. Although the effect of the chemical order on the optical properties is modest, some trends have been identified, especially at low energy. Therefore it would be very desirable to have new photoabsorption experimental data on different phases to verify, if such trends can be detected by experiments and therefore are useful to assess the structures of these elusive systems.

Acknowledgements. This work was supported by Stiftung Beneficentia and by Finanziamento per la Ricerca di Ateneo, FRA 2015 and FRA 2016 of the Università degli Studi di Trieste. S. O. thanks HPC-Europa3 initiative for supporting her stay at Barcelona University during the preparation of the TCCM Master Thesis. Work of L. V. was financed by the Generalitat de Catalunya via a pre-doctoral grant 2018FI-B-00384. L. V., F. V. and K. M. N. acknowledge support from Spanish Ministry of Science and Universities (MICIUN) for the Maria de Maeztu Unit of Excellence MDM-2017-0767 and the Ministry of Economy and Competitiveness (MEC) for the CTQ2015-64618-R funding as well as by the grant 2017SGR13 of the Generalitat de Catalunya and thank the Red Española de Supercomputación for providing computer resources and technical support. L. V. and K. M. N. acknowledge a support by the Spanish/FEDER grant PGC2018-093863-B-C22 and L. V. and F. V. acknowledge support from MICIUN Spanish/FEDER grant RTI2018-095460-B-I00. Finally F. V. is thankful to MEC for his the RYC-2012-10129 Ramón y Cajal research contract. 


\section{REFERENCES}

${ }^{1}$ Burda, C.; Chen, X.; Narayanan, R.; El-Sayed, M. A. Chemistry and Properties of Nanocrystals of Different Shapes. Chem. Rev. 2005, 105, 1025-1102

2 Kozlov, S.M.; Kovács, G.; Ferrando, R.; Neyman, K. M. How to determine accurate chemical ordering in several nanometer large bimetallic crystallites from electronic structure calculations. Chem. Sci. 2015, 6, 3868-3880.

3 Kovács, G.; Kozlov, S. M.; Neyman, K. M. Versatile Optimization of Chemical Ordering in Bimetallic Nanoparticles. J. Phys. Chem. C 2017, 121, 10803-10808.

${ }^{4}$ Ferrando, R.; Jellinek, J.; Johnston, R. L. Nanoalloys: from theory to applications of alloy clusters and nanoparticles. Chem. Rev. 2008, 108, 845-910.

5 Yudanov, I. V.; Neyman, K. M. Stabilization of Au at edges of bimetallic PdAu nanocrystallites. Phys. Chem. Chem. Phys. 2010, 12, 5094-5100.

6 Baseggio, O.; Fronzoni, G.; Stener, M. A New Time Dependent Density Functional Algorithm for Large Systems and Plasmons in Metal Clusters. J. Chem. Phys. 2015, 143, 024106.

${ }^{7}$ Baseggio, O.; De Vetta, M.; Fronzoni, G.; Stener M.; Fortunelli A. A new Time Dependent Density Functional Method for molecular plasmonics: formalism, implementation and the $\mathrm{Au}_{144}(\mathrm{SH})_{60}$ case study. Int. J. Quantum Chem. 2016, 116, 1603-1611.

${ }^{8}$ Baseggio, O; Toffoli, D.; Fronzoni, G.; Stener, M.; Sementa, L.; Fortunelli, A. Extension of the Time Dependent Density Functional complex polarizability algorithm to circular dichroism: implementation and applications to $\mathrm{Ag}_{8}$ and $\mathrm{Au}_{38}\left(\mathrm{SC}_{2} \mathrm{H}_{4} \mathrm{C}_{6} \mathrm{H}_{5}\right)_{24}$ J. Phys. Chem. C 2016, 120, $24335-24345$.

${ }^{9}$ Baseggio, O.; De Vetta, M.; Fronzoni, G.; Stener, M.; Sementa, L.; Fortunelli A.; Calzolari A. Photoabsorption of icosahedral noble metal clusters: an efficient TDDFT approach to large scale systems J. Phys. Chem. C 2016, 120, 12773-12782.

10 Cottancin, E.; Gaudry, M.; Pellarin, M.; Lermé, J.; Arnaud, L.; Huntzinger, J. R.; Vialle, J. L.; Treilleux, M.; Mélinon, P.; Rousset, J. L.; Broyer, M. Optical properties of mixed clusters: comparative study of Ni/Ag and Pt/Ag clusters Eur. Phys. J. D 2003, 24, 111-114.

11 Barcaro, G.; Sementa, L.; Fortunelli A.; Stener M. Optical properties of Pt and Ag-Pt nanoclusters from TDDFT calculations: plasmon suppression by Pt poisoning J. Phys. Chem. C 2014, 118, 28101-28108.

12 Pirart, J.; Front, A.; Rapetti, D.; Andreazza-Vignolle, C.; Andreazza, P.; Mottet, C.; Ferrando, R. Reversed size-dependent stabilization of ordered nanophases. Nat. Comm. 2019, 10, 1982. 
13 Wojtysiak, S.; Solla-Gullón, J.; Dłuzewski, P.; Kudelski, A. Synthesis of core-shell silverplatinum nanoparticles, improving shell integrity. Colloid. Surf. A Physicochem. Eng. Asp. 2014, $441,178-183$.

14 Wilson, N. M.; Pan, Y.-T.; Shao, Y.-T.; Zuo, J.-M.; Yang, H.; Flaherty, D. W. Direct Synthesis of $\mathrm{H}_{2} \mathrm{O}_{2}$ on AgPt Octahedra ACS Catal. 2018, 8, 2880-2889.

15 Yu, S.; Lou, Q.; Han, K.; Wang, Z.; Zhu, H. Synthesis and electrocatalytic performance of MWCNT-supported Ag@Pt coreeshell nanoparticles for ORR. Int. J. Hydrog. Energy 2012, 37, 13365-13370.

16 Quiroz, J.; Barbosa, E. C. M.; Araujo, T. P.; Fiorio, J. L.; Wang, Y.-C.; Zou, Y.-C.; Mou, T.; Alves, T. V.; de Oliveira, D. C.; Wang, B.; Haigh, S. J.; Rossi, L. M.; Camargo, P. H. C. Controlling Reaction Selectivity over Hybrid Plasmonic Nanocatalysts. Nano Letters 2018, 18, $7289-7297$.

${ }^{17}$ Kresse, G.; Furthmüller, Efficient iterative schemes for ab initio total-energy calculations using a plane-wave basis set. J. Phys. Rev. B 1996, 54, 11169-11186.

18 Perdew, J. P.; Burke, K.; Ernzerhof, M. Generalized Gradient Approximation Made Simple. Phys. Rev. Lett. 1996, 77, 3865-3868.

19 Janthon, P.; Kozlov, S. M.; Viñes, F.; Limtrakul, J.; Illas, F. Establishing the Accuracy of Broadly Used Density Functionals in Describing Bulk Properties of Transition Metals. J. Chem. Theory Comput. 2013, 9, 1631-1640.

20 Janthon, P.; Luo, S.; Kozlov, S. M.; Viñes, F.; Limtrakul, J.; Truhlar, D. G.; Illas, F. Bulk properties of transition metals. J. Chem. Theory Comput. 2014, 10, 3832-3839.

21 Vega, L.; Ruvireta, J.; Viñes, F.; Illas, F. Jacob's Ladder as Sketched by Escher: Assessing the Performance of Broadly Used Density Functionals on Transition Metal Surface Properties. $J$.

Chem. Theory Comput. 2018, 14, 395-403.

22 Methfessel, M.; Paxton, A. T. Phys. Rev. B 1989, 40, 3616-3621.

23 Viñes, F.; Illas, F. Neyman, K. Angew. Chem. Int. Ed. 2007, 119, 7224-7227.

24 Gross, E. K. U.; Kohn, W. Time-Dependent Density-Functional Theory. Adv. Quantum Chem. 1990, 21, 255-291.

25 Van Leeuwen, R.; Baerends, E. J. Exchange-correlation potential with correct asymptotic behavior. Phys. Rev. A 1994, 49, 2421-2431.

26 Gritsenko, O. V.; Schipper, P. R. T.; Baerends, E. J. Approximation of the exchangecorrelation Kohn-Sham potential with a statistical average of different orbital model potentials. Chem. Phys. Lett. 1999, 302, 199-207. 
27 van Lenthe, E.; Baerends, E. J.; Snijders, J. G. Relativistic regular two-component Hamiltonians. J. Chem. Phys. 1993, 99, 4597-4610.

${ }^{28}$ Durante, N; Fortunelli, A.; Broyer, M.; Stener, M. Optical Properties of Au Nanoclusters from TD-DFT Calculations, J. Phys. Chem. C 2011, 115, 6277-6282.

29 Sementa, L.; Barcaro, G.; Baseggio, O.; De Vetta, M.; Dass, A.; Aprà, E.; Stener, M.;

Fortunelli, A. Ligand-Enhanced Optical Response of Gold Nanomolecules and its Fragment Projection Analysis: the Case of $\mathrm{Au}_{30}(\mathrm{SR})_{18 .}$. J. Phys. Chem. C 2017, 121, 10832-10842.

30 Theivendran, S.; Chang, L.; Mukherjee, A.; Sementa, L.; Stener, M.; Fortunelli, A.; Dass, A. Principles of Optical Spectroscopy of Aromatic Alloy Nanomolecules: $\mathrm{Au}_{36-\mathrm{x}} \mathrm{Ag}_{\mathrm{x}}(\mathrm{SPh}-\mathrm{tBu})_{24} J$. Phys. Chem. C 2018, 122, 4524-4531.

${ }^{31}$ Ferrando, R.; Fortunelli, A.; Rossi, G. Quantum Effects on the Structure of Pure and Binary Metallic Nanoclusters. Phys. Rev. B: Condens. Matter Mater. Phys. 2005, 72, 085449 (1-9). 32 Yudanov, I. V.; Neyman, K. M. Stabilization of $\mathrm{Au}$ at Edges of Bimetallic PdAu Nanocrystallites. Phys. Chem. Chem. Phys. 2010, 12, 5094-5100. 


\section{Captions to Figures.}

Figure 1. Structures with the lowest-energy chemical orderings of $\mathrm{Ag}_{87} \mathrm{Pt}_{29}, \mathrm{Ag}_{99} \mathrm{Pt}_{17}, \mathrm{Ag}_{105} \mathrm{Pt}_{35}$, $\mathrm{Ag}_{120} \mathrm{Pt}_{20}, \mathrm{Ag}_{151} \mathrm{Pt}_{50}$, and $\mathrm{Ag}_{172} \mathrm{Pt}_{29}$ particles. $\mathrm{Pt}$ and $\mathrm{Ag}$ atoms are shown as cyan and pink spheres, respectively.

Figure 2. Optical spectra of the closed@shell AgPt particles.

Figure 3. Optical spectra of AgPt particles with same composition Ag:Pt, but increasing size.

Figure 4. Optical spectra of AgPt particles with same size but increasing Pt concentration.

Figure 5. Fragment analysis of the absorption spectra of the closed@shell AgPt particles.

Figure 6. ICM-OS analysis of $\left[\mathrm{Ag}_{87} \mathrm{Pt}_{29}\right]^{-}$cluster at $5.16 \mathrm{eV}$ and $4.32 \mathrm{eV}$.

Figure 7. Partial DOS analysis of the AgPt clusters. The dotted line represents the Fermi energy.

Figure 8. Structures of core-shell and layered $\mathrm{Ag}_{158} \mathrm{Pt}_{43}$ and $\mathrm{Ag}_{161} \mathrm{Pt}_{40}$ particles. $\mathrm{Pt}$ and $\mathrm{Ag}$ atoms are shown as cyan and pink spheres, respectively.

Figure 9. Optical spectra of the core@shell and layered $\mathrm{Ag}_{158} \mathrm{Pt}_{43}$ and $\mathrm{Ag}_{161} \mathrm{Pt}_{40}$ particles. 


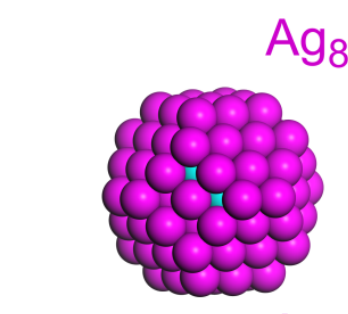

$\mathrm{Ag}_{105} \mathrm{Pt}_{35}$

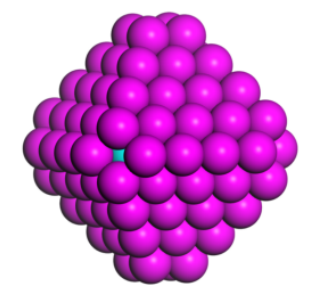

$\mathrm{Ag}_{151} \mathrm{Pt}_{50}$

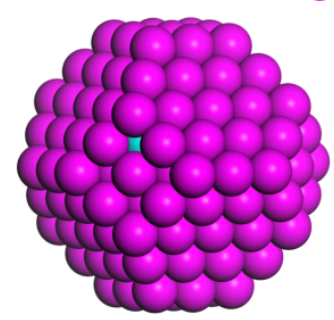

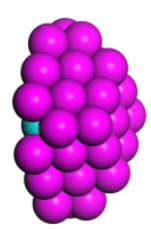

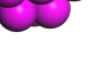
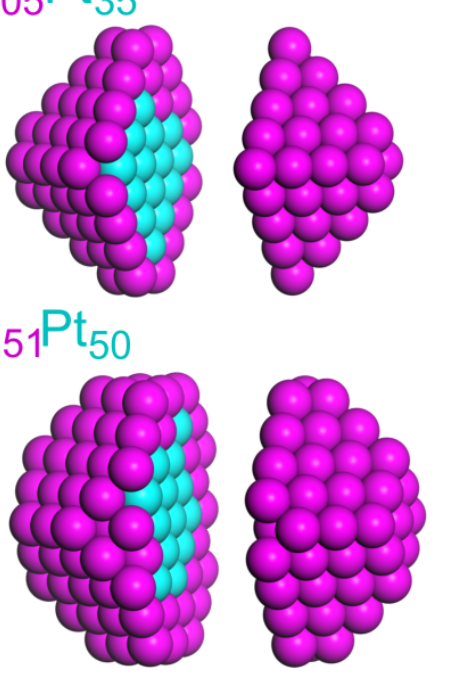

$\mathrm{Ag}_{99} \mathrm{Pt}_{17}$

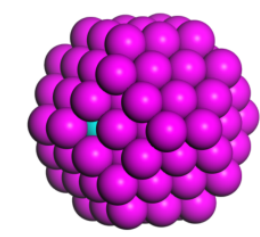

$\mathrm{Ag}_{120} \mathrm{Pt}_{20}$
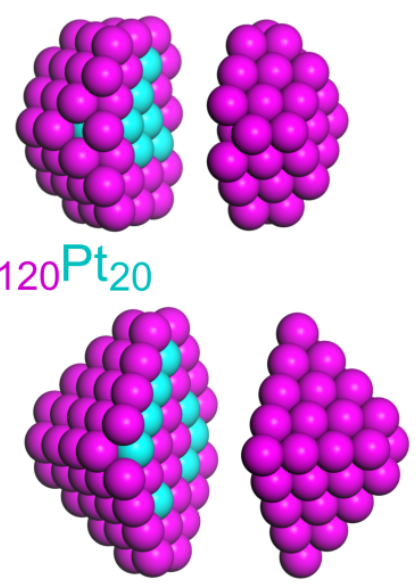

$\mathrm{Ag}_{172} \mathrm{Pt}_{29}$
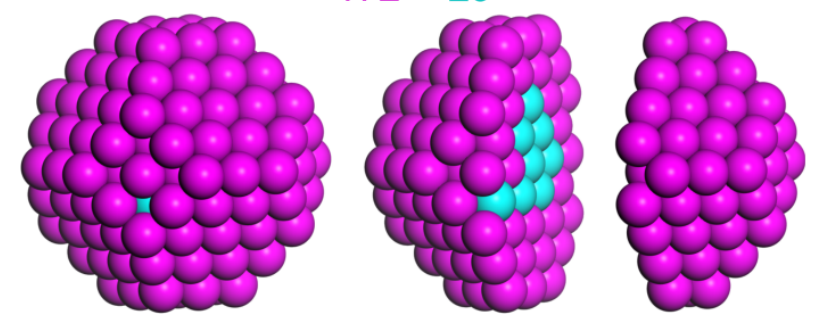

Figure 1 
$\left[\mathrm{Ag}_{87} \mathrm{Pt}_{29}\right]^{-}$

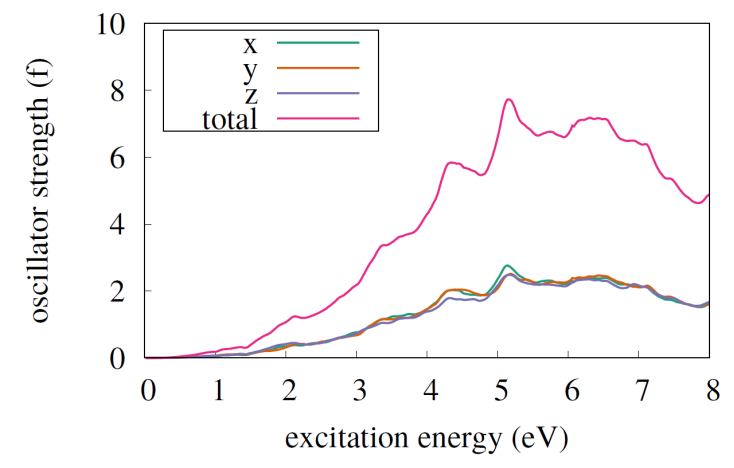

$\left[\mathrm{Ag}_{105} \mathrm{Pt}_{35}\right]^{3+}$

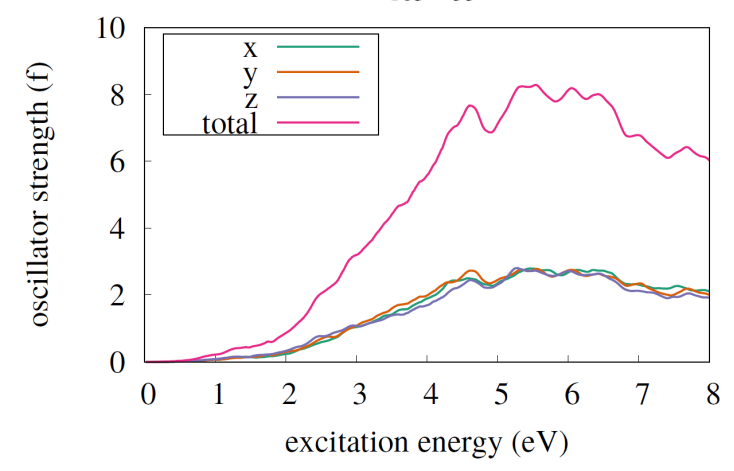

$\left[\mathrm{Ag}_{151} \mathrm{Pt}_{50}\right]^{+}$

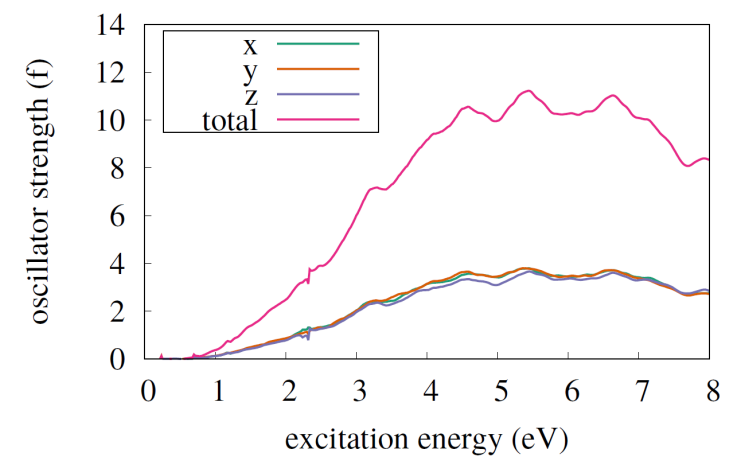

$\left[\mathrm{Ag}_{99} \mathrm{Pt}_{17}\right]^{+}$

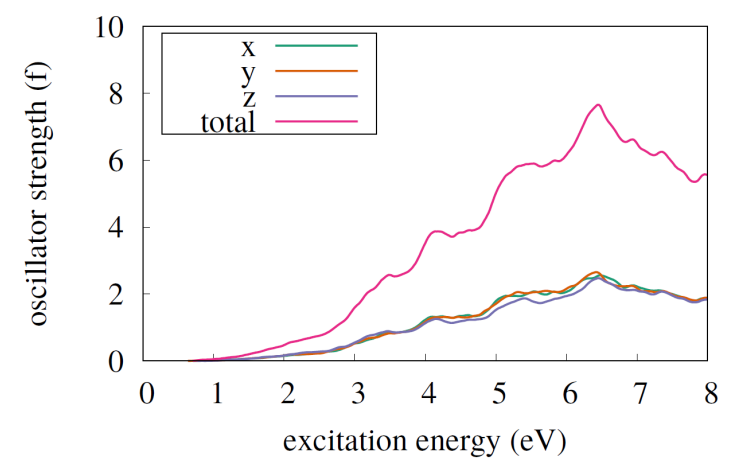

$\left[\mathrm{Ag}_{120} \mathrm{Pt}_{20}\right]$

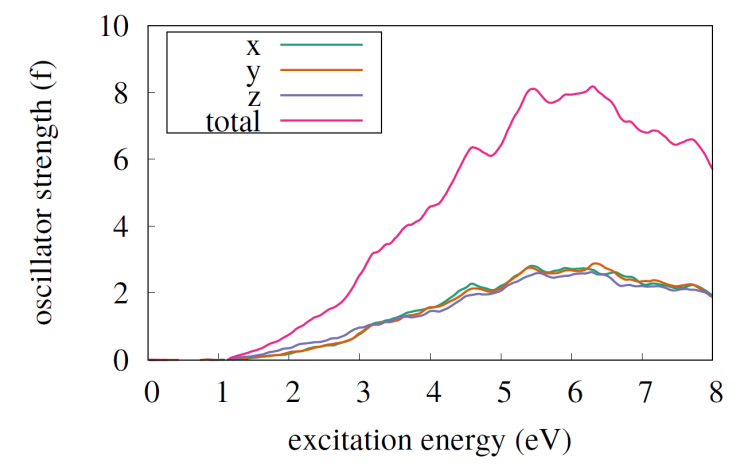

$\left[\mathrm{Ag}_{172} \mathrm{Pt}_{29}\right]^{4+}$

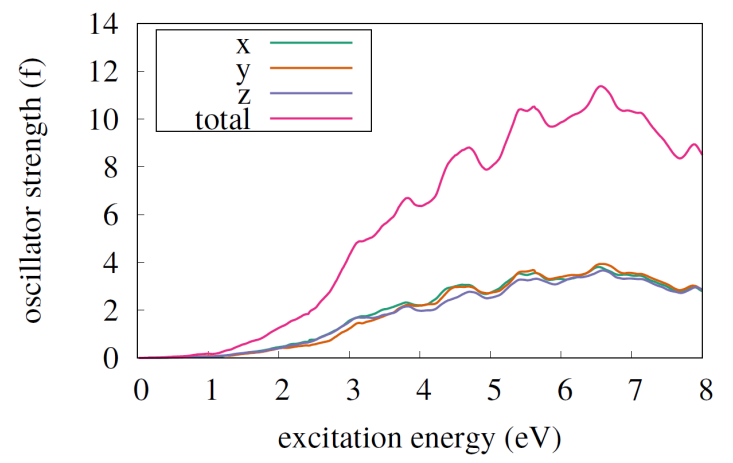

Figure 2 

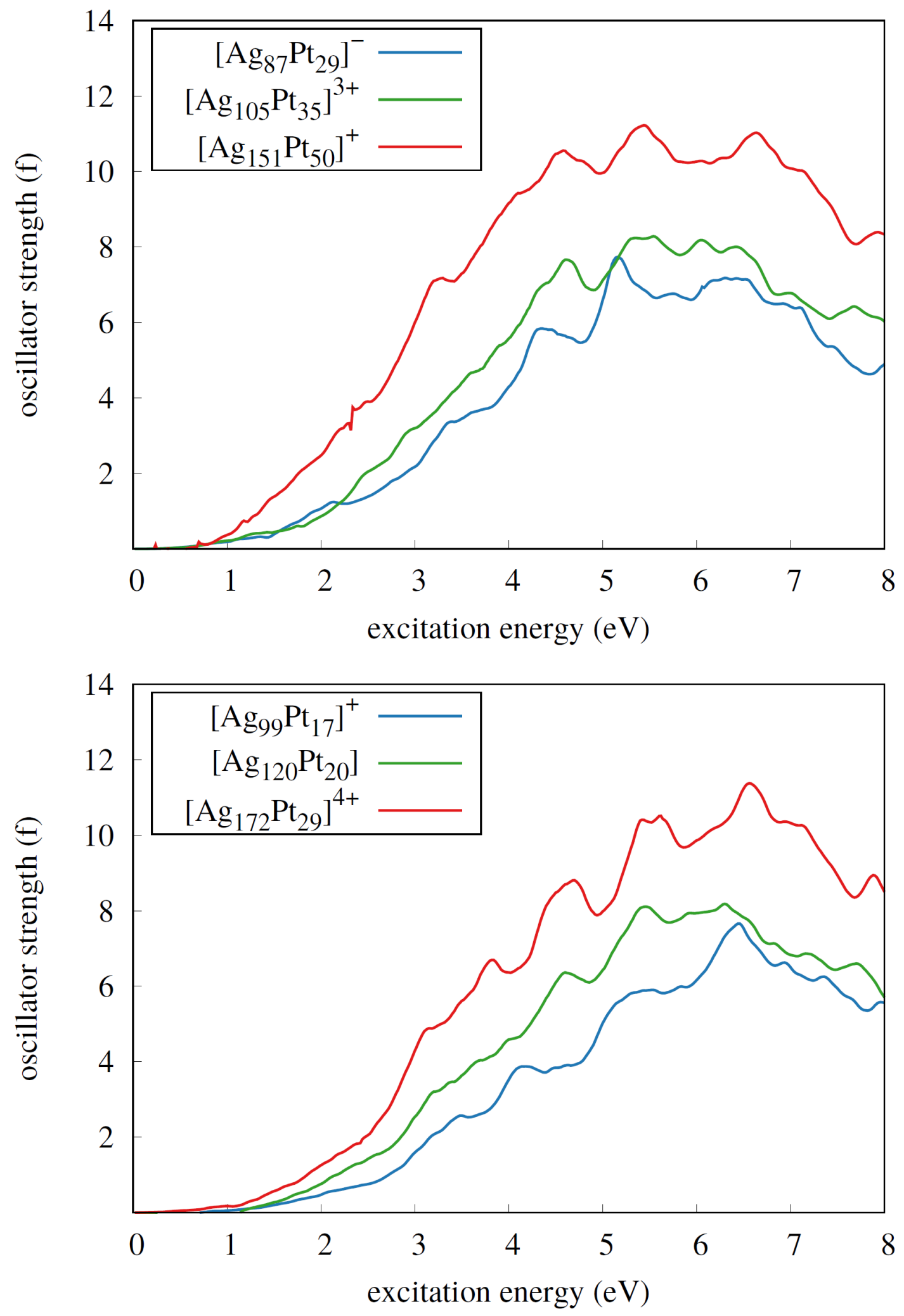

Figure 3 

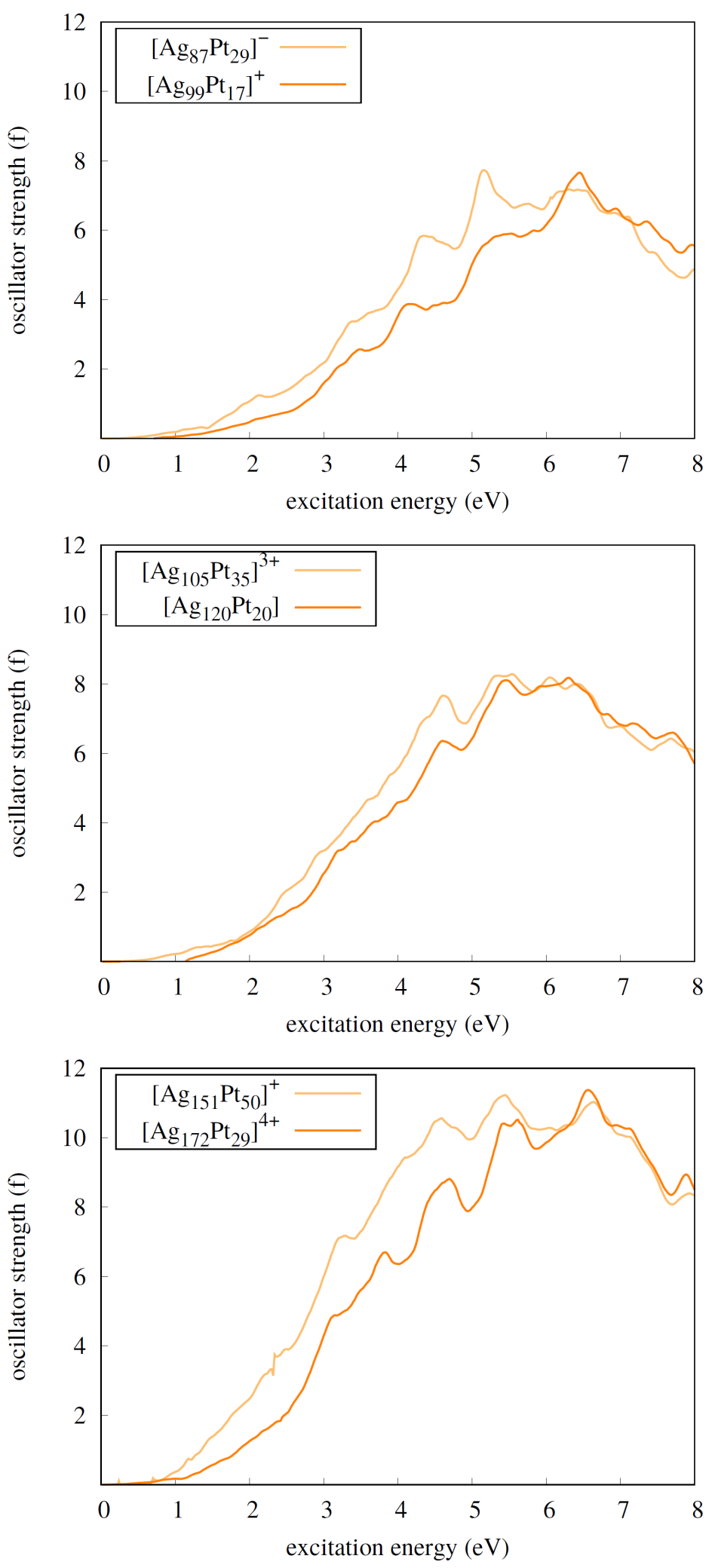

Figure 4 

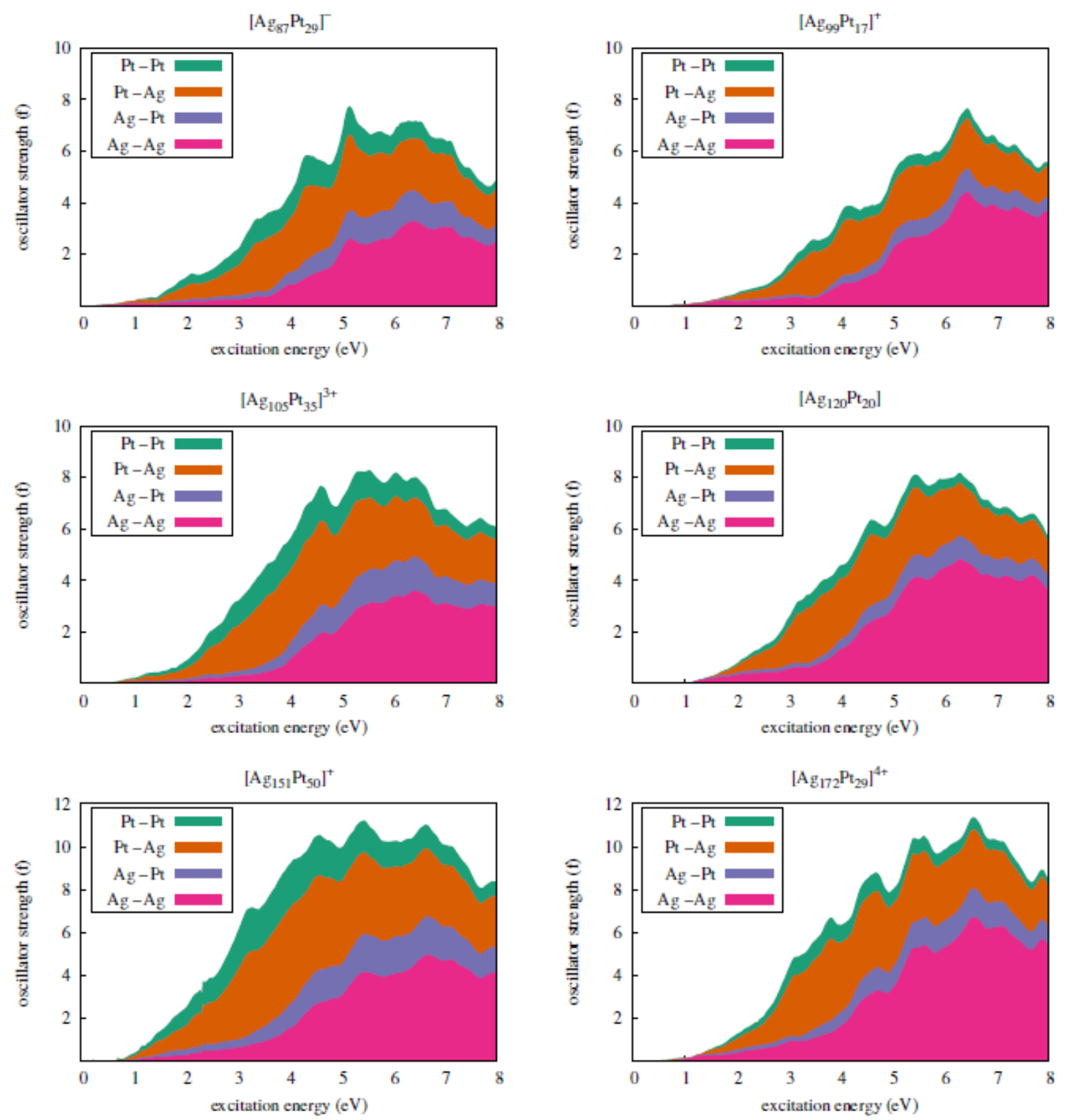

Figure 5 

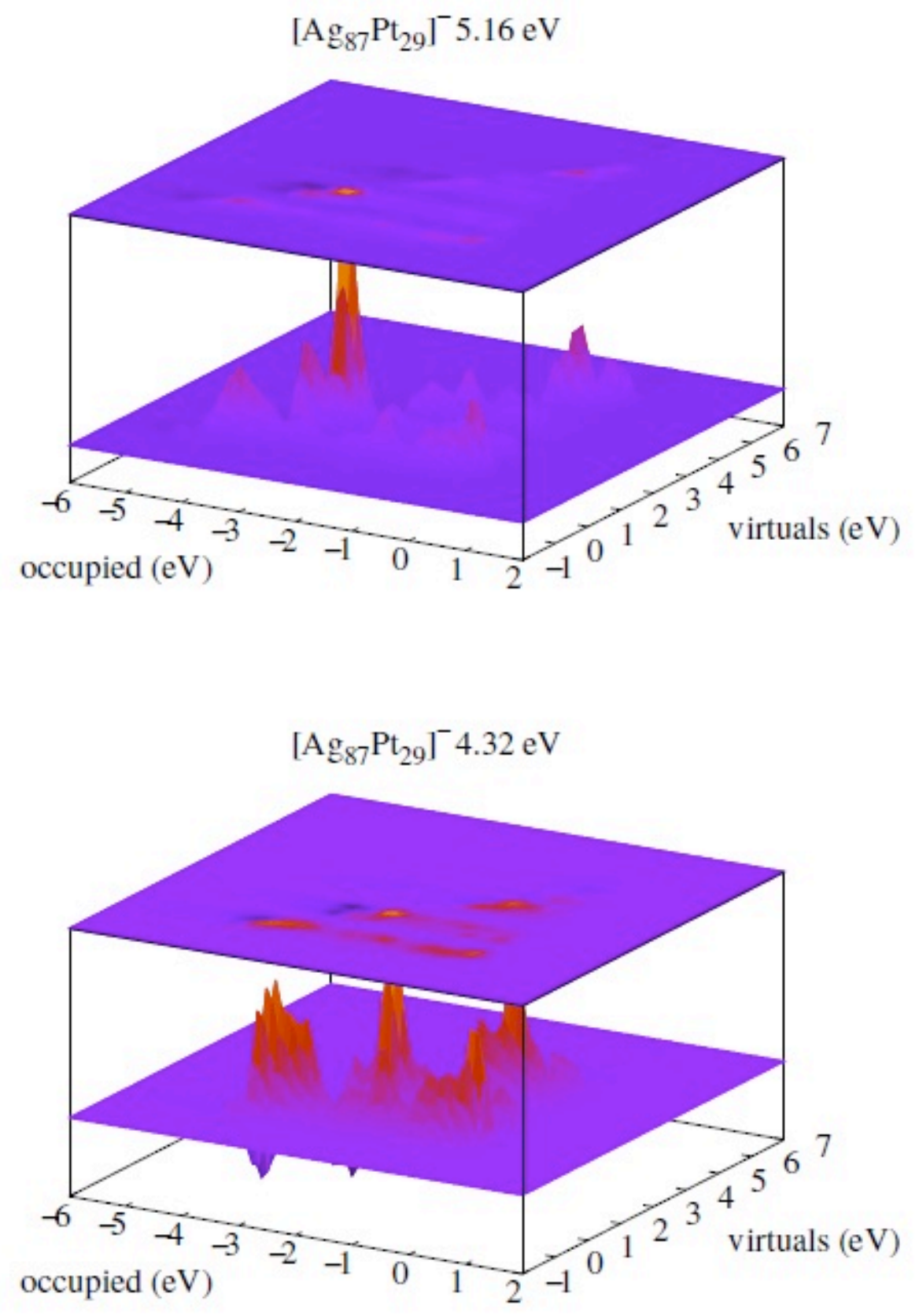

Figure 6 

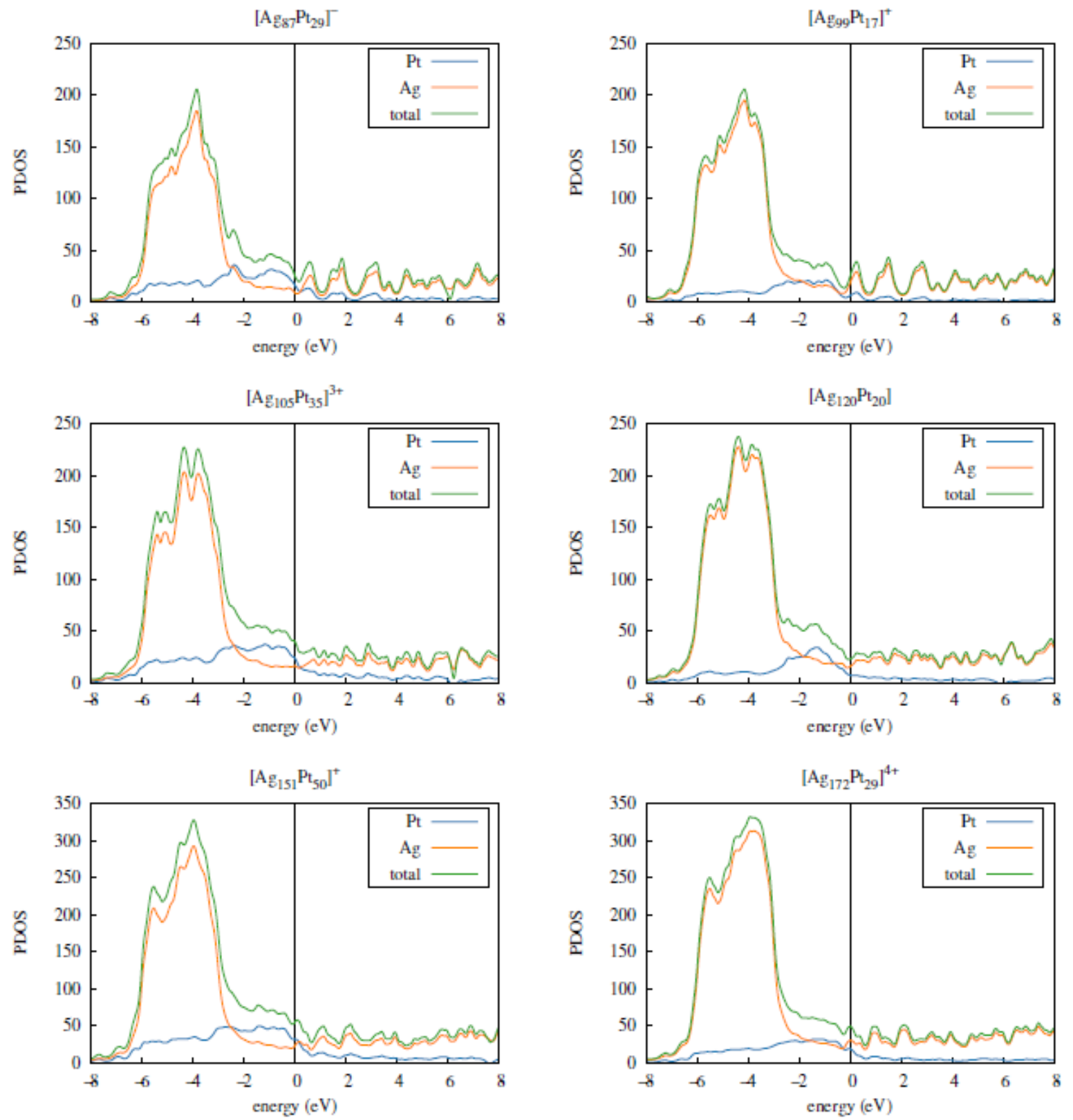

Figure 3.10: Partial DOS of AgPt clusters. The dotted line represents the Fermi energy.

Figure 7 

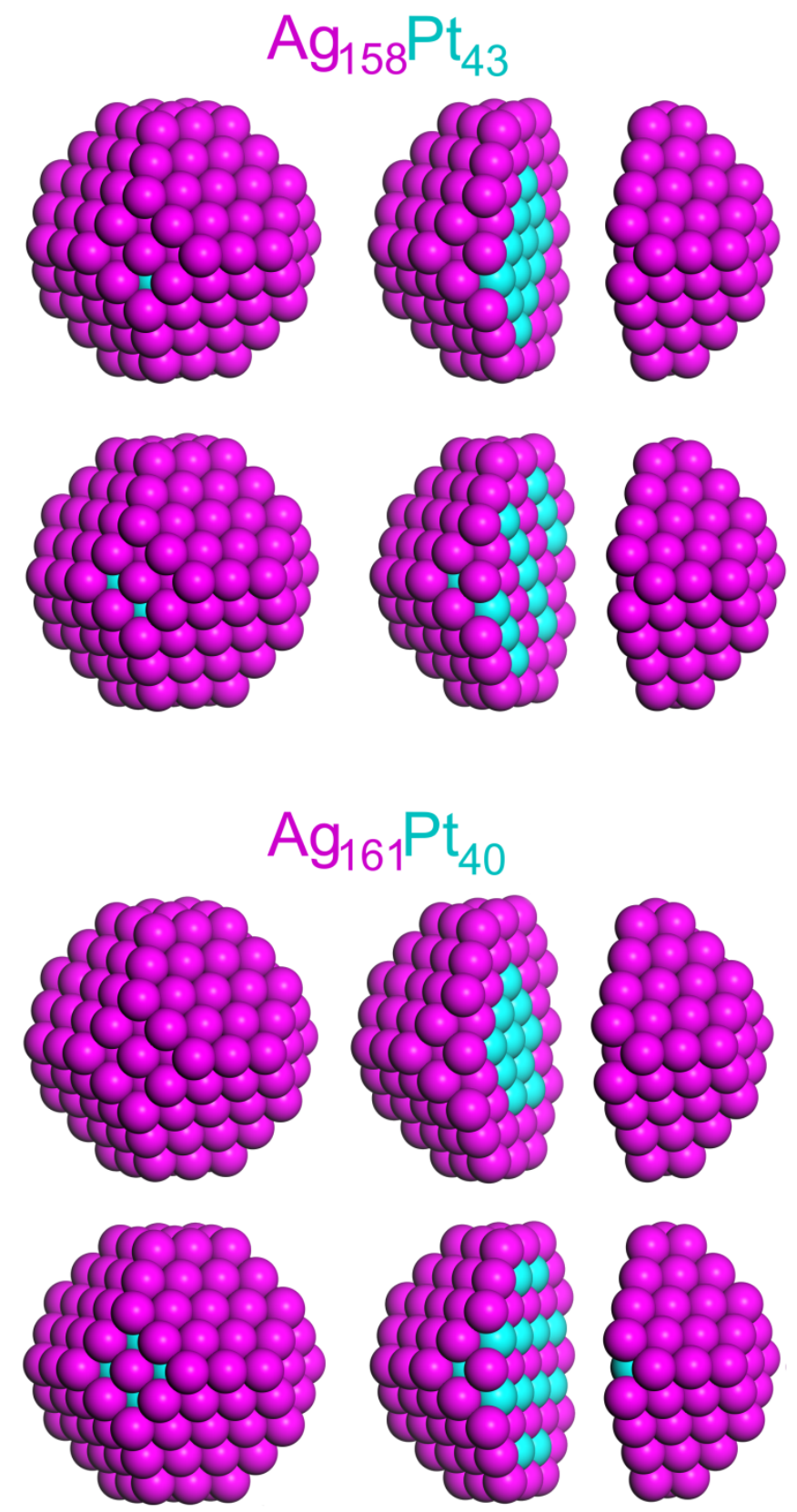

Figure 8 


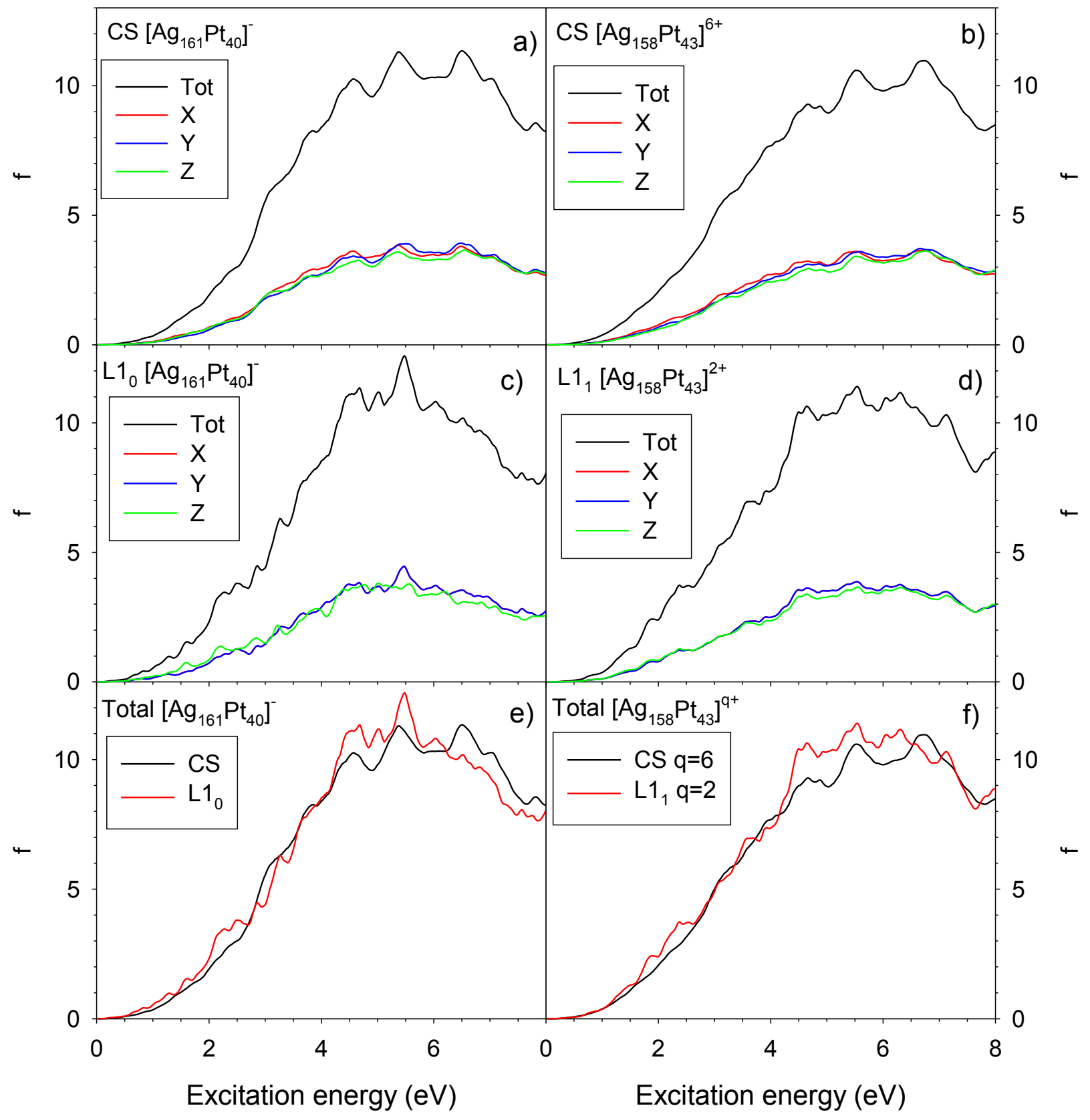

Figure 9 


\title{
Supporting information
}

\section{Optical properties and chemical ordering of Ag-Pt nanoalloys: a computational study}

\author{
Sofia Olobardi ${ }^{1}$, Alessandro Fortunelli ${ }^{2}$, Mauro Stener ${ }^{1 *}$ \\ Francesc Viñes ${ }^{3}$, Lorena Vega $^{3}$ and Konstantin M. Neyman ${ }^{3,4 *}$ \\ ${ }^{1}$ Dipartimento di Scienze Chimiche e Farmaceutiche, Università di Trieste, via L. Giorgieri 1, I- \\ 34127, Trieste, Italy \\ ${ }^{2}$ CNR-ICCOM \& IPCF, Consiglio Nazionale delle Ricerche, via G. Moruzzi 1, 56124, Pisa, \\ Italy \\ ${ }^{3}$ Departament de Ciència dels Materials i Química Física \& Institut de Química Teòrica i \\ Computacional, Universitat de Barcelona, 08028 Barcelona, Spain \\ ${ }^{4}$ ICREA (Institució Catalana de Recerca i Estudis Avançats), 08010 Barcelona, Spain
}

\section{Contents:}

Figure S1. Optical spectra of the closed-shell $\left[\mathrm{Ag}_{99} \mathrm{Pt}_{17}\right]^{+}$and $\left[\mathrm{Ag}_{99} \mathrm{Pt}_{17}\right]^{-}$clusters.

Figure S2. Optical spectra of the closed-shell $\left[\mathrm{Ag}_{116}\right]^{4+},\left[\mathrm{Ag}_{140}\right]^{2+}$, and $\left[\mathrm{Ag}_{201}\right]^{-}$clusters.

Figure S3. ICM-OS analysis of $\left[\mathrm{Ag}_{87} \mathrm{Pt}_{29}\right]^{-},\left[\mathrm{Ag}_{99} \mathrm{Pt}_{17}\right]^{+}$, and $\left[\mathrm{Ag}_{105} \mathrm{Pt}_{35}\right]^{3+}$ clusters.

Figure S4. ICM-OS analysis of $\left[\mathrm{Ag}_{120} \mathrm{Pt}_{20}\right],\left[\mathrm{Ag}_{151} \mathrm{Pt}_{50}\right]^{+}$, and $\left[\mathrm{Ag}_{172} \mathrm{Pt}_{29}\right]^{4+}$ clusters.

Figure S5. Partial DOS analysis of the core shell and layered $\mathrm{Ag}_{158} \mathrm{Pt}_{43}$ and $\mathrm{Ag}_{161} \mathrm{Pt}_{40}$ clusters. The dotted line represents the Fermi energy.

Figure S6. Fragment analysis of the absorption spectra of the core@shell and layered $\mathrm{Ag}_{158} \mathrm{Pt}_{43}$ and $\mathrm{Ag}_{161} \mathrm{Pt}_{40}$ clusters.

Figure S7. ICM-OS analysis of the core@shell and layered $\mathrm{Ag}_{158} \mathrm{Pt}_{43}$ clusters. 


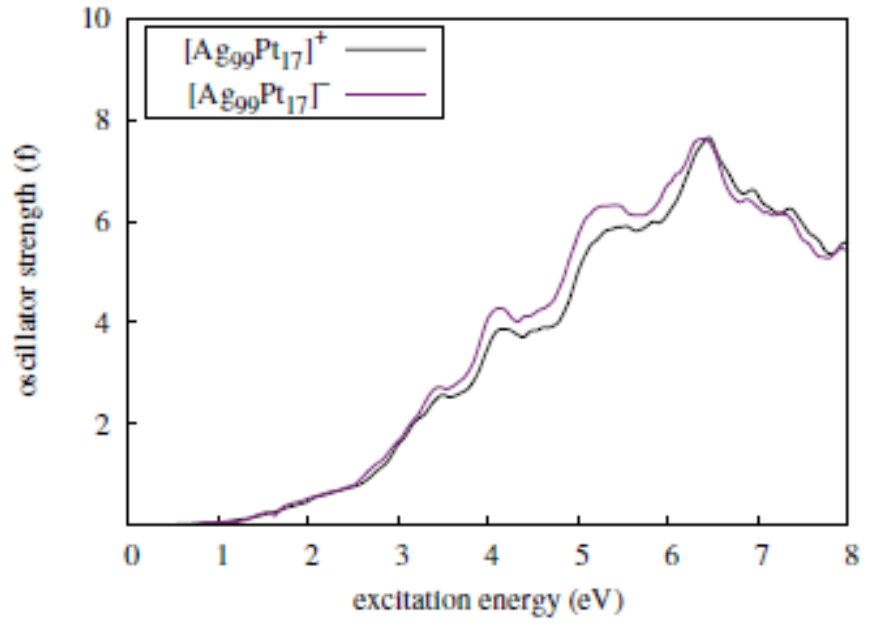

Figure S1 


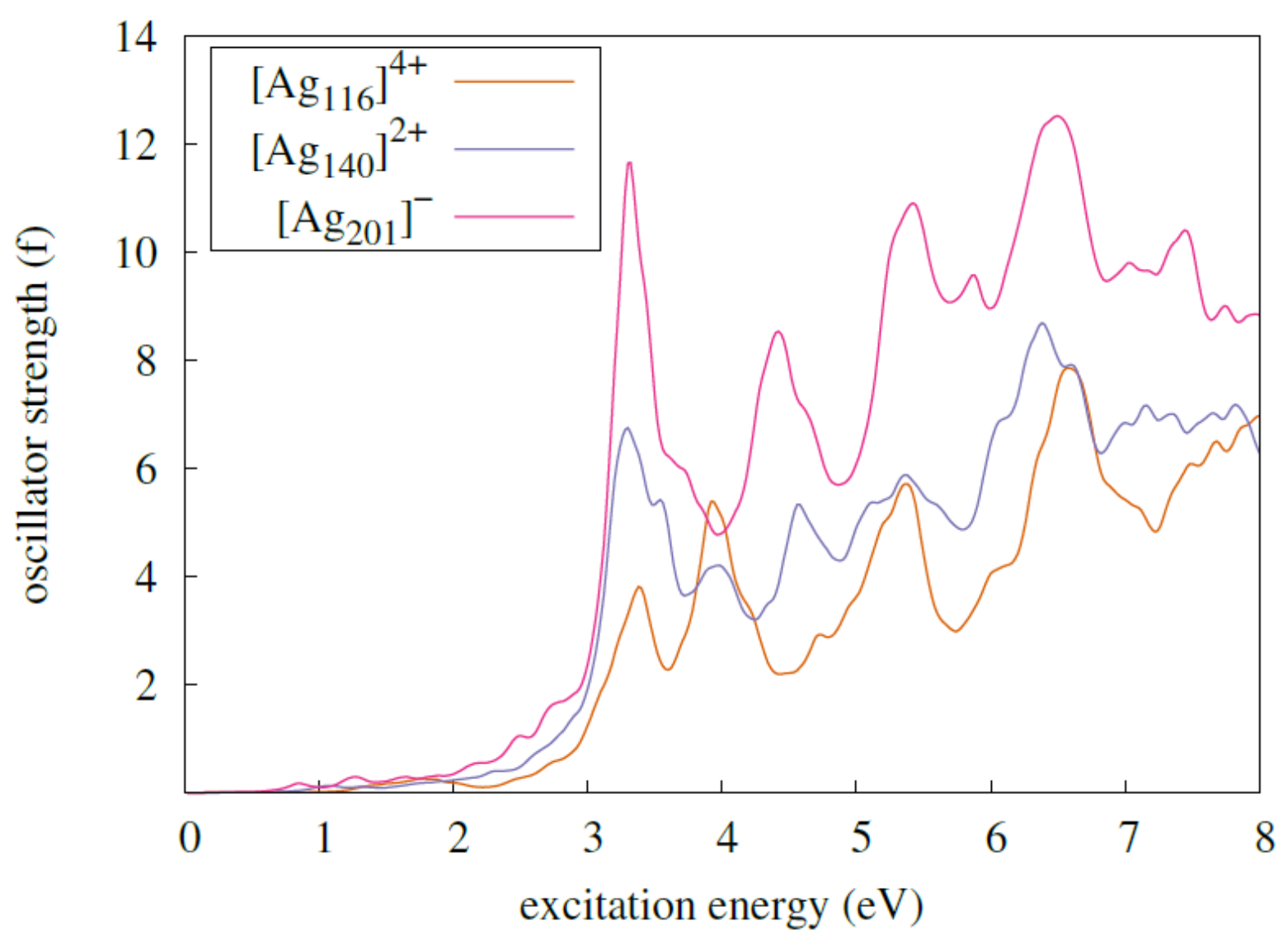

Figure S2 
$\left[\mathrm{Agg}_{87} \mathrm{Pt}_{29}\right]^{-} 4.32 \mathrm{eV}(\mathrm{z})$

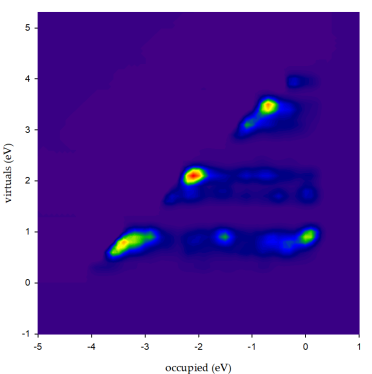

$\left[\mathrm{Ag}_{99} \mathrm{Pt}_{17}\right]^{+} 5.40 \mathrm{eV}(\mathrm{z})$

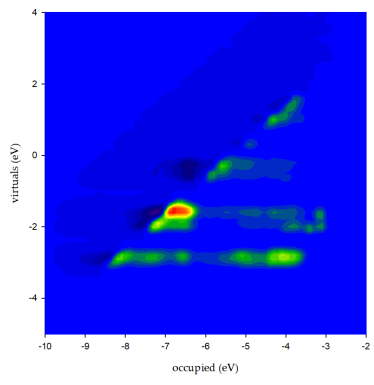

$\left[\mathrm{Ag}_{105} \mathrm{Pt}{ }^{35}\right]^{3+} 4.62 \mathrm{eV}(\mathrm{z})$

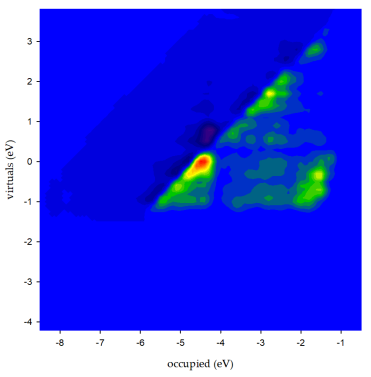

$\left[\mathrm{Ag}_{87} \mathrm{Pt}_{29}\right]^{-5} 5.16 \mathrm{eV}(\mathrm{z})$

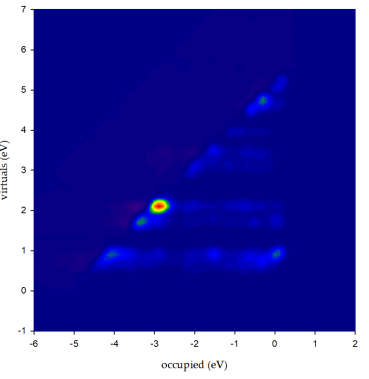

$\left[\mathrm{Ag}_{99} \mathrm{Pt}_{17}\right]^{+} 6.44 \mathrm{eV}(\mathrm{z})$

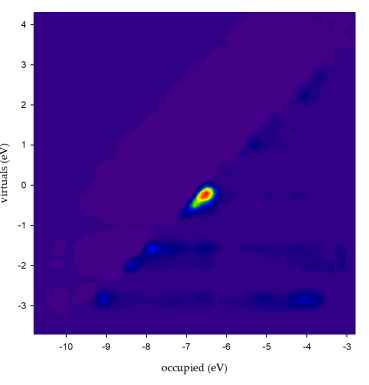

$\left[\mathrm{Ag}_{105} \mathrm{Pt}_{35}\right]^{3+} 5.28 \mathrm{eV}(\mathrm{z})$

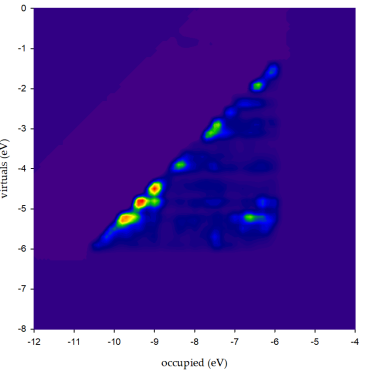

Figure S3 
$\mathrm{Ag}_{120} \mathrm{Pt}_{20} 4.70 \mathrm{eV}(\mathrm{z})$

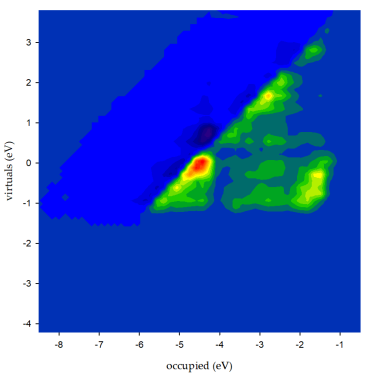

$\left[\mathrm{Ag}_{151} \mathrm{Pt}_{50}\right]^{+} 4.58 \mathrm{eV}(\mathrm{z})$

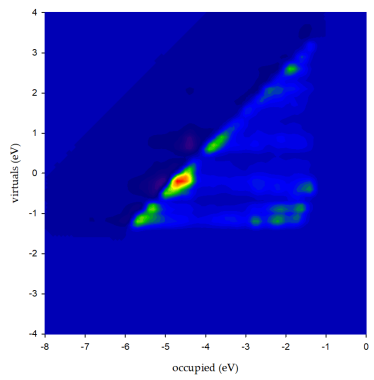

$\left[\mathrm{Ag}_{172} \mathrm{Pt}_{29}\right]^{4+} 4.70 \mathrm{eV}(\mathrm{z})$

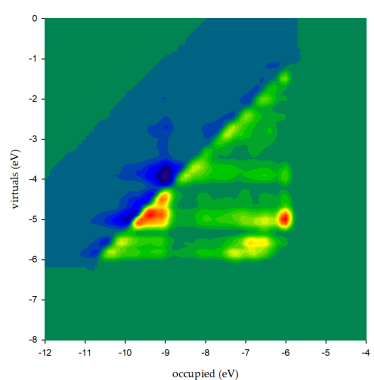

$\mathrm{Ag}_{120} \mathrm{Pt}_{20} 5.54 \mathrm{eV}(\mathrm{z})$

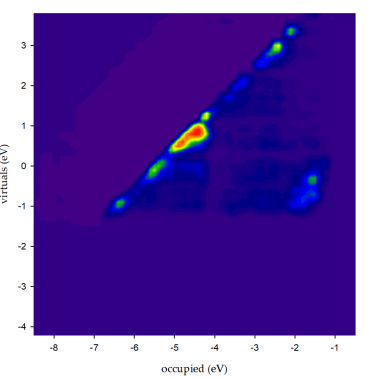

$\left[\mathrm{Ag}_{151} \mathrm{Pt}_{50}\right]^{+} 5.46 \mathrm{eV}(\mathrm{z})$

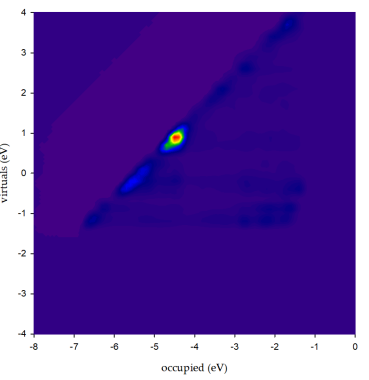

$\left[\mathrm{Ag}_{172} \mathrm{Pt}_{29}\right]^{4+} 5.74 \mathrm{eV}(\mathrm{z})$

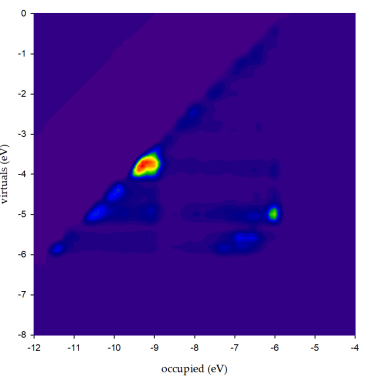

Figure S4 


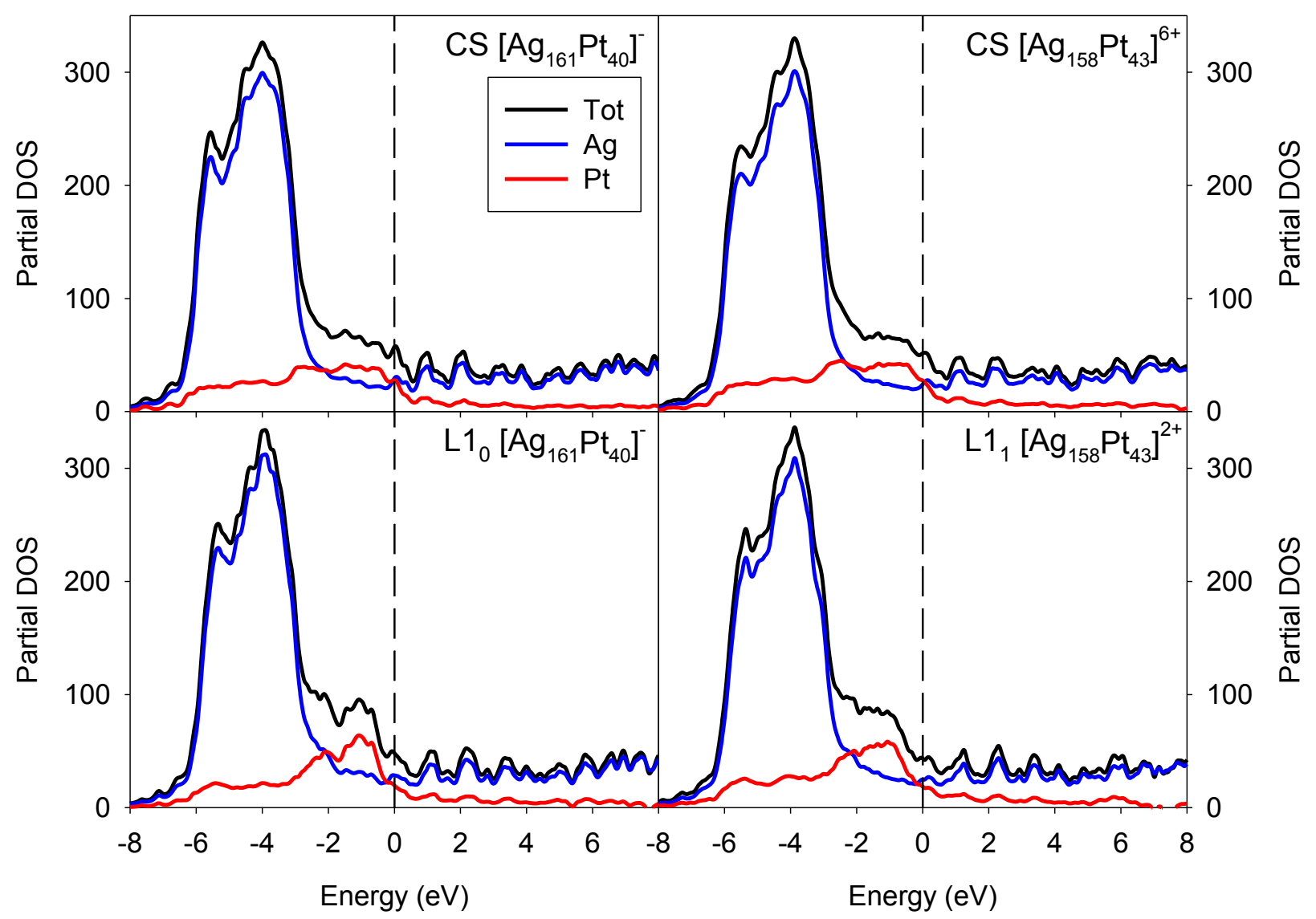

Figure S5 


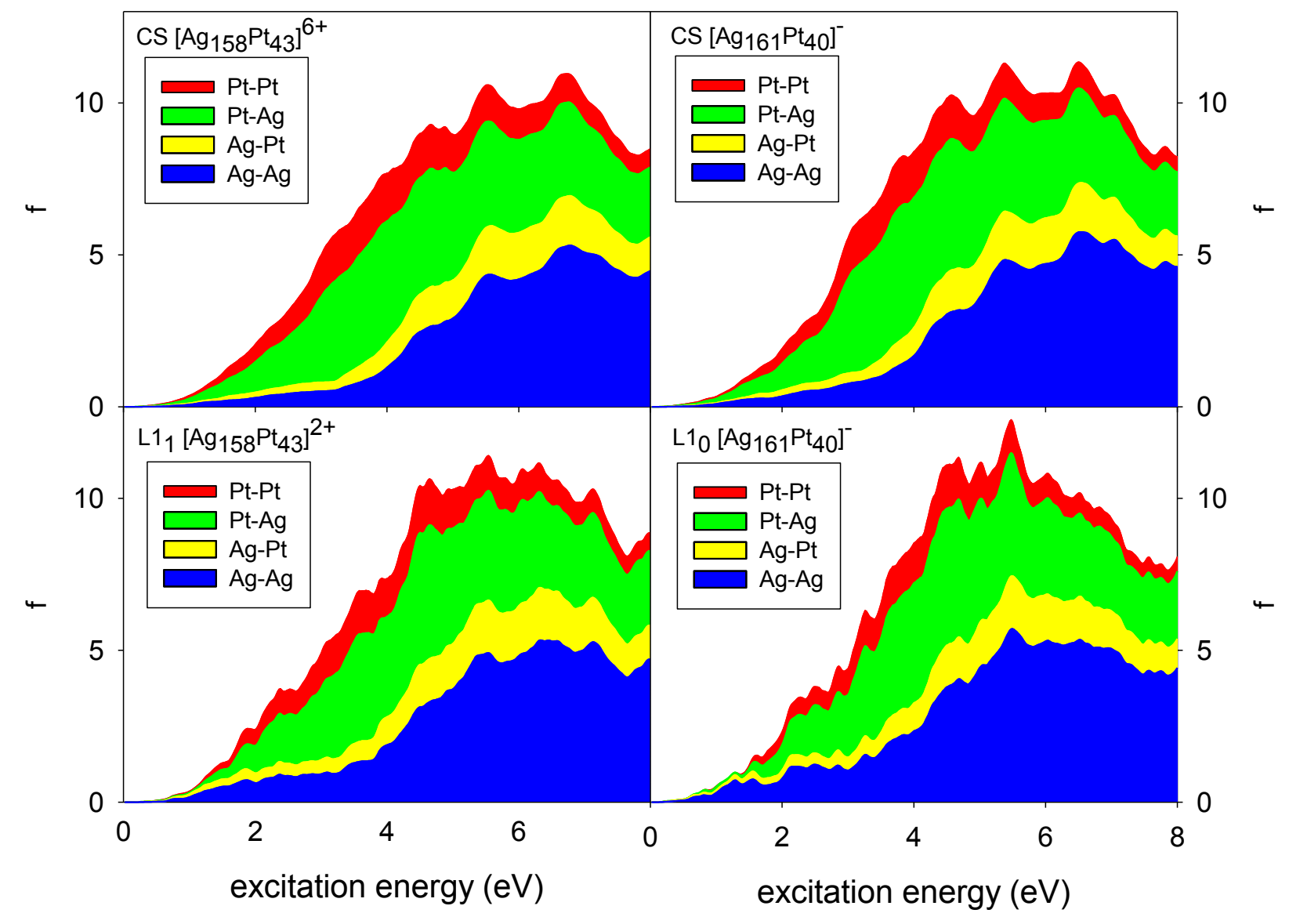

Figure S6 

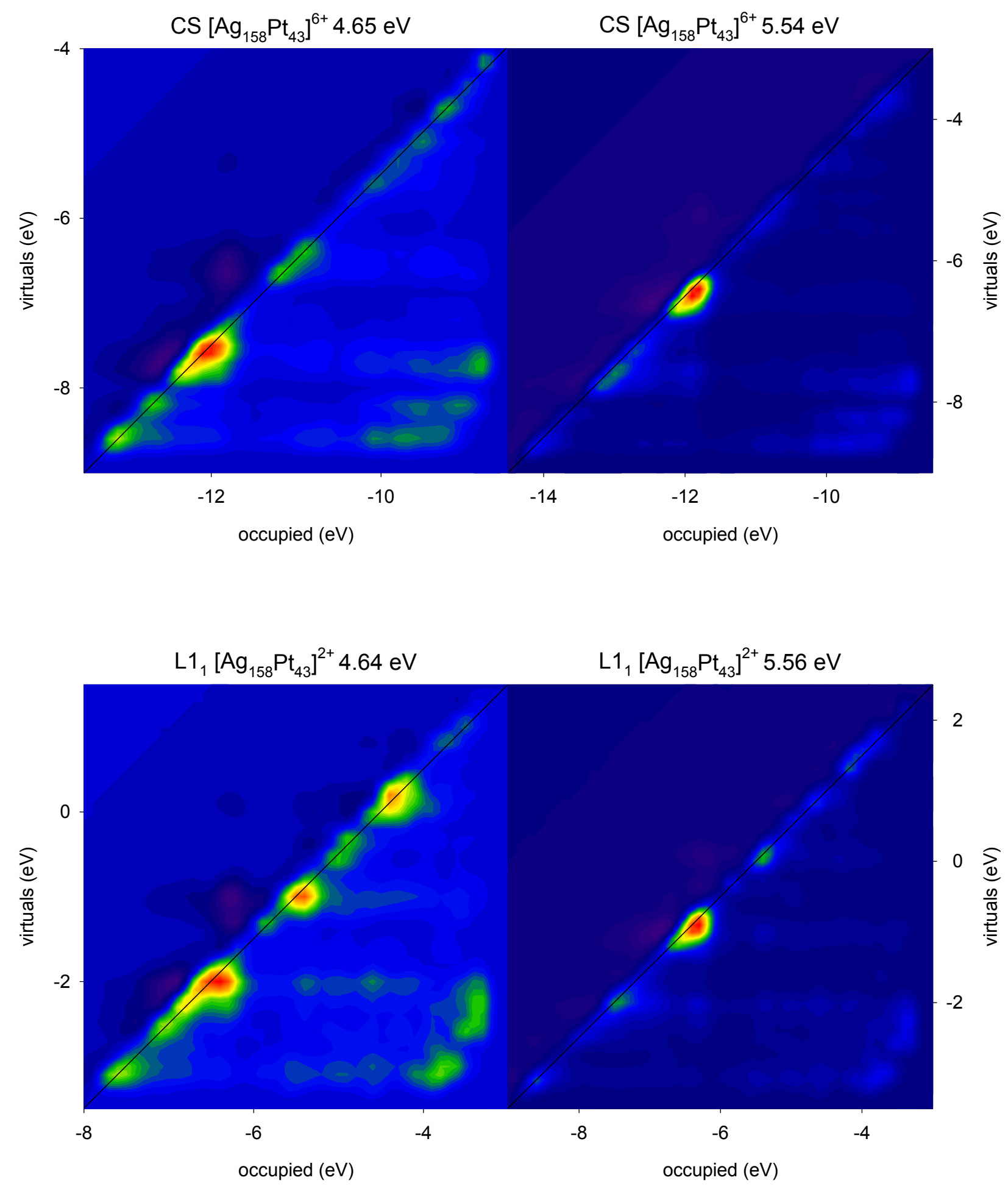

Figure S7 